\title{
CLASH: PRECISE NEW CONSTRAINTS ON THE MASS PROFILE OF THE GALAXY CLUSTER A2261
}

\author{
Dan Coe $^{1}$, Keitchi Umetsu ${ }^{2}$, Adi Zitrin ${ }^{3}$, Megan Donahue ${ }^{4}$, Elinor Medezinski ${ }^{5}$, Marc Postman ${ }^{1}$, \\ Mauricio Carrasco $^{6,7}$, Timo Anguita ${ }^{6,8}$, Margaret J. Geller ${ }^{9}$, Kenneth J. Rines ${ }^{9}, 10$, Antonaldo Diaferio ${ }^{11,12}$, \\ Michael J. Kurtz ${ }^{9}$, Larry Bradley ${ }^{1}$, Anton Koekemoer ${ }^{1}$, Wei Zheng ${ }^{5}$, Mario Nonino ${ }^{13}$, Alberto Molino ${ }^{14}$, \\ Andisheh Mahdavi ${ }^{15}$, Doron Lemze ${ }^{5}$, Leopoldo Infante $^{6}$, SARa Ogaz $^{1}$, Peter Melchior $^{16}$, Ole Host $^{17}$, Holland Ford $^{5}$, \\ Claudio Grillo $^{18}$, Piero Rosati ${ }^{7}$, Yolanda Jiménez-Teja ${ }^{14}$, John Moustakas ${ }^{19}$, Tom Broadhurst ${ }^{20,21}$, Begoña Ascaso ${ }^{14}$, \\ Ofer Lahav $^{17}$, Matthias BartelmanN ${ }^{3}$, Narciso Benítez ${ }^{14}$, Rychard Bouwens $^{22}$, Or Graur ${ }^{23}$, Genevieve Graves ${ }^{24}$, \\ Saurabh Jha $^{25}$, Stephanie Jouvel ${ }^{17}$, Daniel Kelson ${ }^{26}$, Leonidas Moustakas ${ }^{27}$, Dan Maoz ${ }^{23}$, Massimo Meneghetti ${ }^{28}$, \\ Julian Merten $^{27}$, Adam Riess ${ }^{1,5}$, Steve Rodney $^{5}$, and Stella Seitz ${ }^{29}$ \\ ${ }^{1}$ Space Telescope Science Institute, 3700 San Martin Drive, Baltimore, MD 21218, USA; DCoe@ STScI.edu \\ ${ }^{2}$ Institute of Astronomy and Astrophysics, Academia Sinica, Taipei, Taiwan \\ ${ }^{3}$ Institut für Theoretische Astrophysik, Zentrum für Astronomie der Universität Heidelberg, Heidelberg, Germany \\ ${ }^{4}$ Department of Physics and Astronomy, Michigan State University, East Lansing, MI, USA \\ ${ }^{5}$ Department of Physics and Astronomy, Johns Hopkins University, Baltimore, MD, USA \\ ${ }^{6}$ Department of Astronomy and Astrophysics, AIUC, Pontificia Universidad Católica de Chile, Santiago, Chile \\ ${ }^{7}$ European Southern Observatory, Garching, Germany \\ ${ }^{8}$ Max-Planck-Institut für Astronomie, Heidelberg, Germany \\ ${ }^{9}$ Smithsonian Astrophysical Observatory, Cambridge, MA, USA \\ ${ }^{10}$ Department of Physics and Astronomy, Western Washington University, Bellingham, WA, USA \\ ${ }^{11}$ Dipartimento di Fisica Generale “Amedeo Avogadro," Università degli Studi di Torino, Turin, Italy \\ ${ }^{12}$ Istituto Nazionale di Fisica Nucleare, Sezione di Torino, Torino, Italy \\ ${ }^{13}$ Istituto Nazionale di Astrofisica, Osservatorio Astronomico di Trieste, Trieste, Italy \\ ${ }^{14}$ Instituto de Astrofísica de Andalucía, Granada, Spain \\ ${ }^{15}$ Department of Physics and Astronomy, San Francisco State University, San Francisco, CA, USA \\ ${ }^{16}$ Center for Cosmology and Astro-Particle Physics, Department of Physics, Ohio State University, Columbus, OH, USA \\ ${ }^{17}$ Department of Physics and Astronomy, University College London, London, UK \\ ${ }^{18}$ Excellence Cluster Universe, Technische Universität München, Garching, Germany \\ ${ }^{19}$ Center for Astrophysics and Space Sciences, University of California, San Diego, CA, USA \\ ${ }^{20}$ Department of Theoretical Physics, University of the Basque Country UPV/EHU, Bilbao, Spain \\ ${ }^{21}$ Ikerbasque, Basque Foundation for Science, Bilbao, Spain \\ ${ }^{22}$ Leiden Observatory, Leiden University, Leiden, The Netherlands \\ ${ }^{23}$ The School of Physics and Astronomy, Tel Aviv University, Tel Aviv, Israel \\ ${ }^{24}$ Department of Astronomy, University of California at Berkeley, Berkeley, CA, USA \\ ${ }^{25}$ Department of Physics and Astronomy, Rutgers University, New Brunswick, NJ, USA \\ ${ }^{26}$ Carnegie Institute for Science, Carnegie Observatories, Pasadena, CA, USA \\ 27 Jet Propulsion Laboratory, California Institute of Technology, La Cañada Flintridge, CA, USA \\ ${ }^{28}$ Istituto Nazionale di Astrofisica, Astronomical Observatory of Bologna, Bologna, Italy \\ ${ }^{29}$ Instituts für Astronomie und Astrophysik, Universitäs-Sternwarte, München, Munchen, Germany \\ Received 2012 January 8; accepted 2012 July 5; published 2012 August 31
}

\begin{abstract}
We precisely constrain the inner mass profile of A2261 $(z=0.225)$ for the first time and determine that this cluster is not "overconcentrated" as found previously, implying a formation time in agreement with $\Lambda$ CDM expectations. These results are based on multiple strong-lensing analyses of new 16-band Hubble Space Telescope imaging obtained as part of the Cluster Lensing and Supernova survey with Hubble. Combining this with revised weaklensing analyses of Subaru wide-field imaging with five-band Subaru + KPNO photometry, we place tight new constraints on the halo virial mass $M_{\mathrm{vir}}=(2.2 \pm 0.2) \times 10^{15} M_{\odot} h_{70}^{-1}$ (within $\left.r_{\mathrm{vir}} \approx 3 \mathrm{Mpc} h_{70}^{-1}\right)$ and concentration $c_{\mathrm{vir}}=6.2 \pm 0.3$ when assuming a spherical halo. This agrees broadly with average $c(M, z)$ predictions from recent $\Lambda \mathrm{CDM}$ simulations, which span $5 \lesssim\langle c\rangle \lesssim 8$. Our most significant systematic uncertainty is halo elongation along the line of sight (LOS). To estimate this, we also derive a mass profile based on archival Chandra X-ray observations and find it to be $\sim 35 \%$ lower than our lensing-derived profile at $r_{2500} \sim 600 \mathrm{kpc}$. Agreement can be achieved by a halo elongated with a $\sim 2: 1$ axis ratio along our LOS. For this elongated halo model, we find $M_{\text {vir }}=(1.7 \pm 0.2) \times 10^{15} M_{\odot} h_{70}^{-1}$ and $c_{\text {vir }}=4.6 \pm 0.2$, placing rough lower limits on these values. The need for halo elongation can be partially obviated by non-thermal pressure support and, perhaps entirely, by systematic errors in the X-ray mass measurements. We estimate the effect of background structures based on MMT/Hectospec spectroscopic redshifts and find that these tend to lower $M_{\text {vir }}$ further by $\sim 7 \%$ and increase $c_{\text {vir }}$ by $\sim 5 \%$.
\end{abstract}

Key words: dark energy - dark matter - galaxies: clusters: individual (Abell 2261) - galaxies: evolution gravitational lensing: strong - gravitational lensing: weak

Online-only material: color figures, machine-readable table 


\section{INTRODUCTION}

Detailed observational constraints of dark matter halos yield important tests to our understanding of structure formation (Natarajan et al. 2007; Borgani \& Kravtsov 2011), the particle nature of dark matter (Clowe et al. 2006; Keeton \& Moustakas 2009), and perhaps the nature of dark energy as well (Grossi \& Springel 2009). Large cluster surveys require precisely determined cluster masses to calibrate their observables and achieve their full potential to constrain cosmology (Henry et al. 2009; Allen et al. 2011).

The galaxy clusters studied best via gravitational lensing appear to have more densely concentrated cores than clusters of similar mass and redshift formed in $\Lambda$ CDM simulations (Broadhurst et al. 2008; Broadhurst \& Barkana 2008; Oguri et al. 2009; Richard et al. 2010; Sereno et al. 2010; Zitrin et al. 2011a; Postman et al. 2012a). (See results from other methods reviewed in Fedeli 2012 and Bhattacharya et al. 2011.) Some of this discrepancy is due to bias, as the clusters selected for the most detailed lensing studies were among the strongest gravitational lenses known. However, it is estimated that even this large $(\sim 50 \%-100 \%)$ bias cannot fully explain the high observed concentrations (Hennawi et al. 2007; Oguri et al. 2009; Meneghetti et al. 2010, 2011; although see Oguri et al. 2012). Baryons, absent from these dark-matter-only simulations, are found to only modify cluster concentrations at the $\lesssim 10 \%$ level (Duffy et al. 2010; Mead et al. 2010; Fedeli 2012).

If confirmed, this result would imply that galaxy clusters formed earlier than their counterparts in simulated $\Lambda \mathrm{CDM}$ universes. We expect that the higher density of the earlier universe would remain imprinted on the cluster cores as we observe them today (e.g., Jing 2000; Bullock et al. 2001; Wechsler et al. 2002; Zhao et al. 2003).

Another possible hint of early cluster formation may be galaxy clusters detected at $z>1$, which are perhaps unexpectedly massive (Stanford et al. 2006; Eisenhardt et al. 2008; Jee et al. 2009; Huang et al. 2009; Rosati et al. 2009; Papovich et al. 2010; Schwope et al. 2010; Gobat et al. 2011; Jee et al. 2011; Foley et al. 2011; Santos et al. 2011, 2012; Planck Collaboration et al. 2011). However, proper applications of extreme value statistics seem to relieve these tensions (Davis et al. 2011; Paranjape et al. 2011; Hotchkiss 2011; Hoyle et al. 2012; Waizmann et al. 2011, 2012a, 2012b; Harrison \& Coles 2011, 2012). Building on results from large $X$-ray surveys (Vikhlinin et al. 2009; Mantz et al. 2010b), large new Sunyaev-Zel'dovich (SZ) surveys will continue to constrain cosmology based on cluster abundance measurements as functions of mass and redshift (Sehgal et al. 2011; Benson et al. 2011).

Mechanisms proposed to explain such early growth include departures from an initially Gaussian spectrum of density fluctuations (e.g., Chongchitnan \& Silk 2011), though we note some such non-Gaussian models can be ruled out based on cosmic X-ray background measurements (Lemze et al. 2009). Early growth may also be explained by higher levels of dark energy in the past. This idea, dubbed early dark energy (Fedeli \& Bartelmann 2007; Sadeh \& Rephaeli 2008; Francis et al. 2009; Grossi \& Springel 2009), would have suppressed structure growth in the early universe, such that clusters would have had to start forming sooner to yield the numbers we observe today. Other dark energy theories with similar implications have also been proposed (e.g., Baldi 2012; Carlesi et al. 2011).

Significant improvements in these observational constraints are being obtained by CLASH, the Cluster Lensing and
Supernova survey with Hubble (Postman et al. 2012a). ${ }^{30}$ CLASH is a 524-orbit multi-cycle treasury Hubble Space Telescope (HST) program to observe 25 galaxy clusters $(0.18<$ $z<0.89$ ) each in 16 filters with the Wide-Field Camera 3 (WFC3; Kimble et al. 2008) and the Advanced Camera for Surveys (ACS; Ford et al. 2003) over the course of three years (HST cycles 18-20). Importantly, 20 CLASH clusters were $\mathrm{X}$-ray selected to be massive and relatively relaxed. This avoids the strong bias toward high concentrations in previously wellstudied clusters selected for their lensing strength.

Abell 2261 (hereafter A2261) was observed as part of the CLASH program. It has a redshift of $z=0.2249$ as measured by Crawford et al. (1995) and refined by Rines et al. (2010).

Weak-lensing (WL) analyses of ground-based imaging of A2261 (Umetsu et al. 2009; Okabe et al. 2010) yielded concentration measurements of $c_{\mathrm{vir}} \sim 6$ or $\sim 10$, with the broad range attributed to measurement uncertainties, the details of the analysis method used, and perhaps subject to uncertainty due to massive background structures identified at $z \sim 0.5$. The latter value $\left(c_{\mathrm{vir}} \sim 10\right)$ would be significantly higher than predicted for an average relaxed cluster of A2261's mass and redshift, $c_{\text {vir }} \sim 5$ from Duffy et al. (2008), although an analysis of more recent simulations yields a much higher prediction, $c_{\mathrm{vir}} \sim 8.5$ (Prada et al. 2012). The WL measurements had overlapping uncertainties, but a preliminary strong-lensing (SL) measurement of the Einstein radius $\left(R_{E} \approx 40^{\prime \prime}\right.$ for a background source at $\left.z_{s}=1.5\right)$ supported the larger value with smaller uncertainties: $c_{\text {vir }}=11 \pm 2$ (Umetsu et al. 2009). This result was also included in Oguri et al. (2009) as 1 of 10 well-studied clusters, all of which had higher than predicted concentrations.

In this work, we revisit both the strong- and weak-lens modeling. Our deep 16-band HST imaging reveals strongly lensed (multiply imaged) galaxies all undetected in the previous $H S T$ imaging (0.5-orbit WFPC2 F606W) and allows us to derive robust and precise photometric redshifts for these arcs, a key ingredient for our mass model of the cluster core.

Detailed SL analysis is required to accurately and precisely measure the inner mass profile and concentration of A2261. By probing the mass profile over a combined two decades of radius, joint analysis of SL plus WL yields significantly higher precision measurements of cluster virial masses and concentrations than either method alone (Meneghetti et al. 2010).

This paper is organized as follows. We describe our HST (Section 2) and MMT spectroscopic (Section 3) observations followed by our strong-lens mass modeling (Section 4). We then introduce our ground-based imaging and WL analyses (Section 5) and derive joint SL + WL constraints (Section 6). We constrain halo triaxiality from joint lensing $+\mathrm{X}$-ray constraints in Section 7 and, finally, compare our mass profile with results from simulations in Section 8. The formation time of A2261 is discussed in a broader context including other observational probes in Section 9, and we summarize our conclusions in Section 10.

Where necessary to calculate distances, etc., we assume a concordance $\Lambda \mathrm{CDM}$ cosmology with $h=0.7, \Omega_{m}=0.3$,

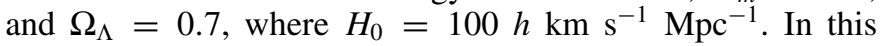
cosmology, at A2261's redshift of $z=0.225,1^{\prime \prime} \approx 3.59 \mathrm{kpc}$ $h_{70}^{-1} \approx 2.51 \mathrm{kpc} h^{-1}$, where $h=0.7 h_{70}$.

Furthermore at this redshift, the cluster virial radius is defined as that which contains an average overdensity of $\Delta_{c} \approx 115$ times critical, where $\Delta_{c} \approx 18 \pi^{2}-82 \Omega_{\Lambda}(z)-39 \Omega_{\Lambda}^{2}(z)$ based

\footnotetext{
${ }^{30} \mathrm{http}: / /$ www.stsci.edu/ postman/CLASH
} 
Table 1

CLASH HST Observations of the Core of A2261

\begin{tabular}{|c|c|c|c|}
\hline $\begin{array}{l}\text { Camera/ } \\
\text { Channel }\end{array}$ & $\begin{array}{c}\text { Filter } \\
\text { Element }\end{array}$ & $\begin{array}{c}H S T \\
\text { Orbits }\end{array}$ & $\begin{array}{c}\text { Exposure Time } \\
\text { (s) }\end{array}$ \\
\hline WFC3/UVIS & F225W & 1.5 & 3671 \\
\hline WFC3/UVIS & F275W & 1.5 & 3745 \\
\hline WFC3/UVIS & F336W & 1.0 & 2408 \\
\hline WFC3/UVIS & F390W & 1.0 & 2456 \\
\hline ACS/WFC & F435W & 1.0 & 2077 \\
\hline $\mathrm{ACS} / \mathrm{WFC}$ & F475W & 1.0 & 2064 \\
\hline $\mathrm{ACS} / \mathrm{WFC}$ & F606W & 1.0 & 2057 \\
\hline ACS/WFC & F625W & 1.0 & 2064 \\
\hline ACS/WFC & F775W & 1.0 & 2072 \\
\hline $\mathrm{ACS} / \mathrm{WFC}$ & F814W & 2.0 & 4099 \\
\hline ACS/WFC & F850LP & 2.0 & 4148 \\
\hline WFC3/IR & F105W & 1.0 & 2814 \\
\hline WFC3/IR & F110W & 1.0 & 2514 \\
\hline WFC3/IR & F125W & 1.0 & 2514 \\
\hline WFC3/IR & F140W & 1.0 & 2411 \\
\hline WFC3/IR & F160W & 2.0 & 5029 \\
\hline
\end{tabular}

Note. Parallel observations and supernova follow-up observations are not described here or utilized in this work.

on spherical collapse theory (Bryan \& Norman 1998). This definition is also commonly used to characterize simulated halos (e.g., Duffy et al. 2008; Klypin et al. 2011; Prada et al. 2012; Bhattacharya et al. 2011). Virial masses and halo concentrations may be converted to $\Delta_{c}=200$ as used in earlier work (beginning with Navarro et al. 1996) via $c_{\text {vir }} \approx 1.232 c_{200}+0.189$ and, for the concentrations found here, $M_{\mathrm{vir}} \sim 1.2 M_{200}($ Coe 2010).

\section{HST OBSERVATIONS}

We observed A2261 (R.A. $=17^{\mathrm{h}} 22^{\mathrm{m}} 27^{\mathrm{s}} .2$, decl. $=+32^{\circ} 07^{\prime} 57^{\prime \prime}$ [J2000] for the BCG, brightest cluster galaxy) as part of the CLASH program in HST cycle 18 between 2011 March 9 and May 21 to a total depth of 20 orbits in 16 WFC3 and ACS filters, spanning 2000-17000 $\AA$ (Table 1; GO 12066; PI: Postman). The full 20-orbit, 16-filter depth is achieved over a central area of $3.9 \operatorname{arcmin}^{2}$, comfortably including all the strongly lensed galaxies (Section 4). The ACS images cover a wider $13.4 \operatorname{arcmin}^{2}$. The images were processed for debias, flats, superflats, and darks using standard techniques and then co-aligned and combined using drizzle algorithms. See Koekemoer et al. (2011) and Postman et al. (2012a) for details.

In order to better reveal faint lensed images, we modeled and subtracted the BCG light in all 12 ACS+IR filters (see also Postman et al. 2012b). We used the isophote fitting routine, SNUC, which is part of the XVISTA image processing system, to derive two-dimensional models of the bright early-type galaxies in A2261, including the BCG. SNUC is capable of simultaneously obtaining the best nonlinear least-squares fits to the two-dimensional surface brightness distributions in multiple, overlapping galaxies (Lauer 1986). The models were derived independently for each CLASH passband. Fits were performed using concentric isophotes, but the position angles and the ellipticities of the isophotes were left as free parameters. The models were then subtracted from the original image to produce a bright-galaxy-subtracted image.

We used SExtractor (Bertin \& Arnouts 1996) to detect objects and measure their photometry. For arcs eluding this initial detection, we constructed manual apertures that were then forced back into SExtractor using SExSeg (Coe et al. 2006). Isophotal apertures were used as they have been shown to yield robust colors (Benítez et al. 2004).

Based on this photometry, we measured photometric redshifts using Bayesian photometric redshifts (BPZ; Benítez 2000; Benítez et al. 2004; Coe et al. 2006). Spectral energy distribution (SED) templates are redshifted and fit to the observed photometry. A Bayesian analysis tempers the qualities of fit with a prior: the empirical likelihood of redshift as a function of both galaxy magnitude and type (e.g., bright and/or elliptical galaxies are rare at high redshift). Here, we used 11 SED templates originally from PEGASE (Fioc \& Rocca-Volmerange 1997) but strongly recalibrated based on photometry and spectroscopic redshifts of galaxies in the FIREWORKS catalog (Wuyts et al. 2008). In analyses of large data sets with high-quality spectra, these templates yield $\lesssim 1 \%$ outliers and therefore implicitly encompass the full range of metallicities, extinctions, and star formation histories of real galaxies.

It is difficult to quantify our photometric redshift accuracy in A2261, as only seven galaxies with confident spectroscopic redshifts (Section 3) are within the area imaged by $16 \mathrm{HST}$ bands. Future work will quantify this accuracy in the larger CLASH sample.

The color images used in this paper were produced automatically using the publicly available Trilogy software. ${ }^{31}$ Trilogy determines the intensity scaling automatically and independently in each color channel to display faint features without saturating bright features. The scalings are determined based on a sample of the summed images and two input parameters. One sets the output luminosity of "the noise," currently determined as $1 \sigma$ above the sigma-clipped mean. The other parameter sets what fraction of the data (if any) in the sample region should be allowed to saturate. Default values for these parameters $(0.15 \%$ and $0.001 \%$, respectively) work well, but the user is able to adjust them. The scaling is accomplished using the logarithmic function $y=a \log _{10}(k x+1)$ clipped between 0 and 1 , where $a$ and $k$ are constants determined based on the data and desired scaling parameters as described above.

\section{MMT/HECTOSPEC SPECTROSCOPY}

The Hectospec instrument mounted on the $6.5 \mathrm{~m} \mathrm{MMT}$ is a multiobject fiber-fed spectrograph with 300 fibers deployable over a $1^{\circ}$ diameter field (Fabricant et al. 2005). We targeted probable A2261 cluster members based on their proximity to the expected cluster red sequence and proximity to the BCG (K. Rines et al. 2012, in preparation). In addition, all targets were sufficiently bright with magnitudes $16<r^{\prime}<21$ in Sloan Digital Sky Survey (SDSS) DR7 (Abazajian et al. 2009). To observe all targets, we used four pointings each with $3 \times 20$ minute exposures within the Hectospec queue schedule.

Our primary targets were observed in excellent conditions and yielded reliable spectroscopic redshifts $97 \%$ of the time. We also observed secondary targets chosen as brighter objects near the cluster center. These were observed in poor conditions with a lower success rate of $67 \%$. Most of the failed spectroscopic observations were due to scattered light from the several bright stars in the field, including HD157465 11' from the cluster center and the naked eye star 72 Her, which lies $29^{\prime}$ from the center at the edge of the field.

After processing and reducing the spectra, we used the Hectospec pipeline (Mink et al. 2007) based on the IRAF

\footnotetext{
31 http://www.stsci.edu/ dcoe/trilogy/
} 
Table 2

MMT/Hectospec Spectroscopic Redshifts for Galaxies within the Subaru FOV

\begin{tabular}{lcccc}
\hline \hline $\begin{array}{l}\text { R.A. } \\
(\mathrm{J} 2000 \mathrm{deg})\end{array}$ & $\begin{array}{c}\text { Decl. } \\
(\mathrm{J} 2000 \mathrm{deg})\end{array}$ & Redshift & $\begin{array}{c}\text { Redshift } \\
\text { Uncertainty }\end{array}$ & $\begin{array}{c}\text { Cluster } \\
\text { Member? }\end{array}$ \\
\hline 260.29303 & 32.07600 & 0.22096 & 0.00017 & 0 \\
260.30228 & 32.26368 & 0.38051 & 0.00020 & 0 \\
260.32202 & 32.27190 & 0.13698 & 0.00007 & 0 \\
260.32373 & 32.22027 & 0.11367 & 0.00009 & 0 \\
260.34888 & 32.04460 & 0.15224 & 0.00008 & 0 \\
\hline
\end{tabular}

Notes. ${ }^{a}$ Based on dynamical analysis of the cluster caustics.

(This table is available in its entirety in a machine-readable form in the online journal. A portion is shown here for guidance regarding its form and content.)

package RVSAO (Kurtz \& Mink 1998) to cross-correlate the spectra with a set of standard Hectospec templates. In total, we obtained high-quality redshifts for $\mathbf{5 7 2}$ galaxies, including 308 within our Subaru analysis region (within 17!8 of the BCG). These redshifts are provided in Table 2. Scattered light from bright stars in this field limits spectroscopy of this system.

Redshift uncertainties are measured for each galaxy individually and have been empirically quantified globally as follows. The SHELS survey (Geller et al. 2005) carried out with Hectospec obtained repeat observations of 1468 galaxies, yielding a mean internal error of $56 \mathrm{~km} \mathrm{~s}^{-1}$ for absorption-line objects and $21 \mathrm{~km} \mathrm{~s}^{-1}$ for emission-line objects (Fabricant et al. 2005). Comparison of spectroscopic redshifts obtained for 379 galaxies in both SHELS and SDSS DR7 yields $\Delta v=$ $10 \mathrm{~km} \mathrm{~s}^{-1} \pm 35 \mathrm{~km} \mathrm{~s}^{-1}$. Note that $\Delta v=30 \mathrm{~km} \mathrm{~s}^{-1}$ corresponds to $\Delta z \approx 0.0001$.

Based on the measured redshifts, we identified cluster members using the caustic technique (e.g., Diaferio 2009). The technique locates two curves, the caustics, in the cluster redshift diagram, namely, the plane of the line-of-sight (LOS) velocities of the galaxies versus their projected clustercentric distances. The caustics are related to the escape velocity from the cluster and define an area of the redshift diagram where most of the cluster members reside. Samples of members identified with the caustic technique are at least $95 \%$ complete and contaminated by interlopers by $10 \%$ at most (A. L. Serra et al. 2012, in preparation). This procedure has been used to identify galaxy members of clusters and groups (e.g., Rines et al. 2005; Rines \& Diaferio 2006, 2010), as well as stellar members of the Milky Way halo (Brown et al. 2010) and of dwarf spheroidals (Serra et al. 2010).

We applied the technique to a sample of 641 galaxies with confident spectroscopic redshifts from Hectospec and SDSS DR7 in the field of A2261 (K. Rines et al. 2012, in preparation). The technique identifies 209 members within $6.6 \mathrm{Mpc} h_{70}^{-1}$ $(\sim 0.5)$ of the cluster center. Within 0.25 , we obtained redshifts for 127 of 307 galaxies toward the bright end of the red sequence $\left(18<r^{\prime}<19.5 ; 1.2<g-r<1.5\right)$ and found 97 to be cluster members.

We note that the caustic technique also locates the cluster center $\sim 1.3 \mathrm{Mpc}\left(\sim 6^{\prime}\right)$ south of the BCG location and at a redshift greater by 0.0017 . This result indicates that the dynamical structure of A2261 might be more complex than expected.

We used cluster members identified here to validate galaxy selections based on broadband photometric colors used in our SL (Section 4.1) and WL analyses (Section 5.5).

\section{STRONG-LENS MASS MODELING OF THE CLUSTER CORE}

We performed three semi-independent SL analyses on the A2261 HST images. We used the method of Zitrin et al. (2009b) to perform the primary SL analysis (Section 4.1), including the identification of multiple-image systems (Section 4.2). These multiple images are all identified for the first time in this work based on our HST imaging and lens modeling. We verified these identifications using the Lenstool ${ }^{32}$ modeling software (Kneib 1993; Jullo et al. 2007), as well as LensPerfect ${ }^{33}$ (Coe et al. 2008, 2010), a "non-parametric" method that does not require the assumption that light traces mass (Section 4.3). Finally, we combined the results from all methods, yielding an average cluster core mass profile with uncertainties (Section 4.4). By utilizing various modeling methods, we captured the true systematic uncertainties more reliably than generally possible using a single method.

\subsection{Primary Strong-lensing Analysis Method}

The Zitrin et al. (2009b) method was adapted from that used in Broadhurst et al. (2005a), reducing the number of free parameters to six, and has been used extensively since (Zitrin et al. 2009a, 2009b, 2010, 2011a, 2011b, 2011c, 2012a, 2012b, 2012c; Zitrin \& Broadhurst 2009; Merten et al. 2011). In this work, as also performed in Umetsu et al. (2012), we add a seventh free parameter, the BCG mass, and we explore this parameter space using Markov Chain Monte Carlo (MCMC). Here, we also introduce an alternative Gaussian convolution kernel to parameterize how light traces mass.

The mass model consists of four components: the BCG, the remaining cluster galaxies, a dark matter halo, and an external shear to account for the combined effects of shear due to structures at larger radius, as well as ellipticity in the mass distribution in the plane of the sky within or around the core. Cluster galaxy light is assumed to approximately trace the dark matter; the latter is modeled as a smoothed version of the former, as described below.

We identified 118 probable cluster galaxies along the "red sequence," which is well isolated in F814W-F475W color-magnitude space. We verified, using additional filters and photometric redshifts, that this selection is robust. We also compared this selection to Hectospec spectroscopic redshifts available for 15 galaxies within the HST FOV. We correctly identified 11 of the 13 cluster members, missing one near the FOV edge and another near the bright star. We incorrectly identified one of the two foreground objects $(z=0.1693)$ as a cluster member as it fell along our red sequence. These three particular misidentifications have a negligible effect on our mass model as they all lie at $R>80^{\prime \prime}$, well outside the SL region where multiple images are formed. Nor do these $\sim 10 \%$ rates of incompleteness and contamination significantly affect our mass profile as evidenced in part by our other analyses (Section 4.4). The cluster members provide a parameterization for the mass model that is not required to be exact but rather provides a starting point that is molded to fit the data.

Each cluster galaxy is modeled as a power-law density profile, its mass scaling with flux observed in F814W. This mass distribution is then smoothed using a two-dimensional polynomial spline or Gaussian to provide a model for the dark matter distribution in the cluster halo. This "smooth" mass

\footnotetext{
32 http://www.oamp.fr/cosmology/lenstool/

33 http://www.its.caltech.edu/ coe/LensPerfect/
} 

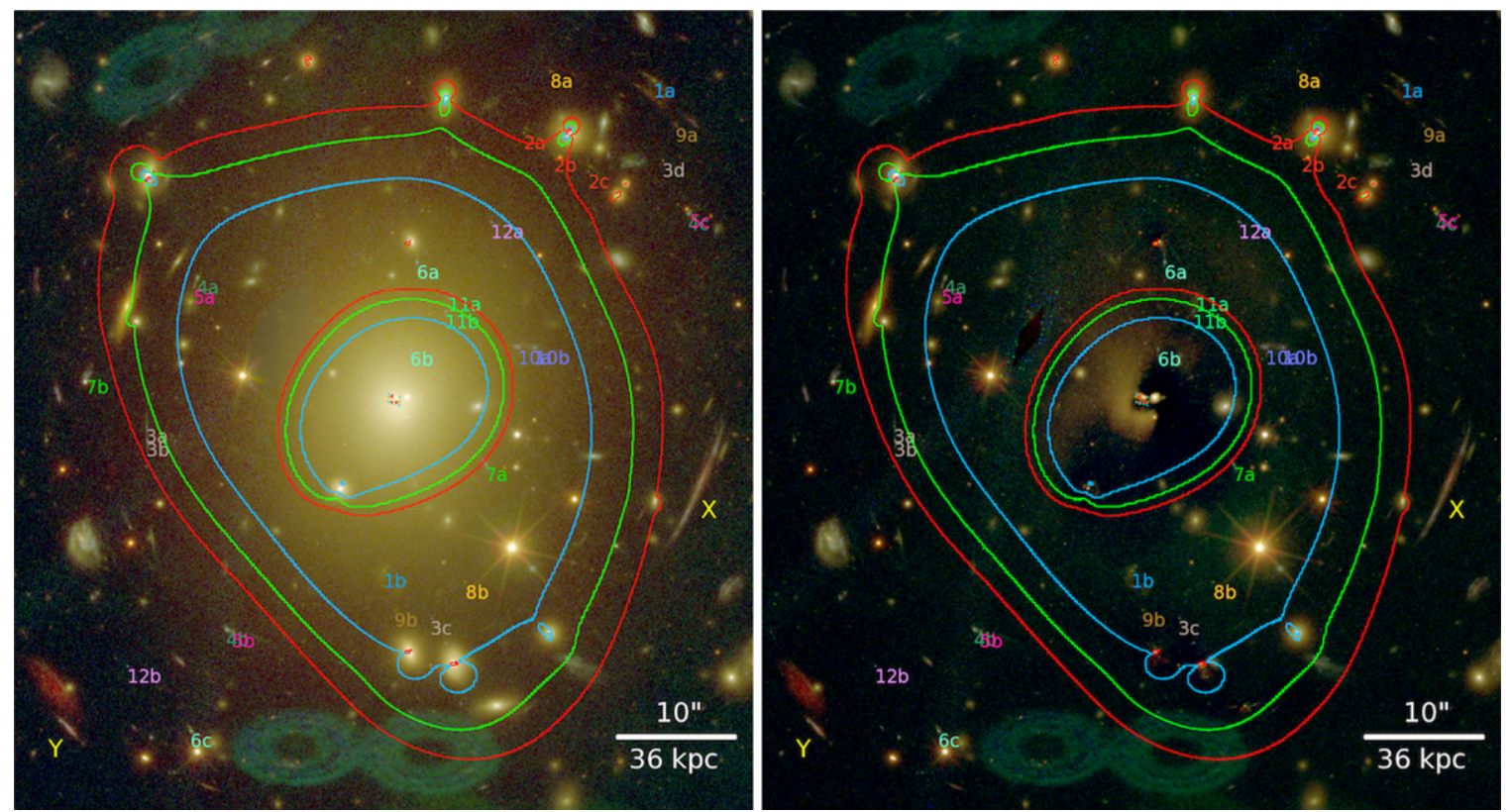

Figure 1. Multiple images of background galaxies strongly lensed by A2261. All were identified in this work based on our deep, multiband HST imaging and lens modeling. Each is located directly above and to the left of its label. Close-ups may be seen in Figure 2. The prominent arc marked "X" is not multiply imaged according to our models (see Figure 4). Lensing critical curves from our primary lens model are plotted for background sources at redshifts $z_{s}=1$ (blue), 2 (green), and 7 (red). These HST ACS+WFC3/IR color images were produced automatically using Trilogy (Section 2), which reveals faint features without saturating bright areas such as the BCG core. Filters were assigned colors as follows: blue $=$ F435W + F475W, green $=$ remaining ACS filters, and red $=$ WFC $3 / \mathrm{IR}$. The green "Figure 8 " patterns are ACS reflection artifacts from a bright star to the SE. North is up and east is left. Left: the diamond-shaped hole in the ACS images was filled in with an IR image tinted yellow to roughly match the color of this portion of the BCG's stellar halo. Right: light has been modeled and subtracted from the BCG and a few other cluster galaxies close to the arcs (Section 2). The residual pattern near the location of the BCG center (aside from the bright knots) is most likely due to a combination of small model artifacts and real asymmetries in the stellar distribution.

(A color version of this figure is available in the online journal.)

component is added to the more "lumpy" (unsmoothed) galaxy component. Finally, an external shear is added.

In all, there are seven free parameters: the mass scalings of both the "smooth" and galaxy components, the BCG mass, the power law of the galaxy density profiles, the degree of the smoothing polynomial (or the Gaussian width), and the amplitude and direction of the external shear. The MCMC routine iterates over lens models to find those that acceptably reproduce the observed positions of the strongly lensed images in the image plane, rather than in the source plane, which can bias solutions toward flatter profiles and higher magnifications unless handled carefully as in Jullo et al. (2007), for example. Details may be found in Zitrin et al. (2009b).

The observational uncertainties of the lensed image positions are on the order of 0.205 , one ACS pixel. This is negligible compared to the scatter on the order of $1^{\prime \prime}$ that may be induced by LOS structures (primarily behind the cluster) plus another $\lesssim 1^{\prime \prime}$ from scatter in the mass-to-light scaling relations of cluster galaxies (Jullo et al. 2010; D'Aloisio \& Natarajan 2012; Host 2012). We adopt a total uncertainty of 1".4 as also used in Zitrin et al. (2012c).

\subsection{Multiple Images of Strongly Lensed Galaxies}

Using this method, we identified 30 multiple images of 12 background galaxies strongly lensed by A2261 (see Figures 1 and 2 and Table 3). These are all identified for the first time in this work. They provide 36 constraints to our mass modeling: $2\left(N_{\text {images }}-N_{\text {systems }}\right)=$ two coordinates $(x, y)$ from each of the 30 multiple images minus 12 for the unknown source positions. (Only relative deflections yield constraints.)
We used an iterative process to identify images and add them to the model, beginning with those that are most confident. Our most confident multiple-image system is the "claw" or U-shaped object, system 1. The distinctive morphology is apparent in both images, including a color gradient best viewed in the IR color images with the BCG subtracted (see Figure 2). Image 1a yields a photo- $z, z \sim 4.4$. The IR flux of image $1 \mathrm{~b}$ appears to be biased a bit high by contaminating light from the BCG (Figure 3), such that the best-fit SED is an early-type galaxy at $z \sim 0.5$. The irregular morphology is not consistent with an early-type galaxy.

Based on this system, we obtained the initial mass model, enabling us to predict the lensed positions of counterimages of other galaxies by delensing them to their putative true source positions and then relensing them with our model. Candidate counterimages were identified as being near the observed position, with the predicted lensed morphology and orientation and consistent colors and photometric redshifts. Observed photometry and SED fits are shown in Figure 3. Our multiple images generally have consistent observed SEDs (allowing for variations in magnification) and thus photo-z's. However, some images yield unreliable photo-z's if they are faint and/or their light is contaminated by a bright nearby cluster member. To date, no spectroscopic redshifts are available for these galaxies strongly lensed by A2261.

We note that our lens models do not predict counterimages for the large prominent arc marked with an " $\mathrm{X}$ " in Figure 1. Instead, they predict a single highly distorted image, as observed (see Figure 4). We measure its photometric redshift to be $z=1.19_{-0.02}^{+0.05}(95 \%$ C.L.). If it was at a slightly higher redshift 

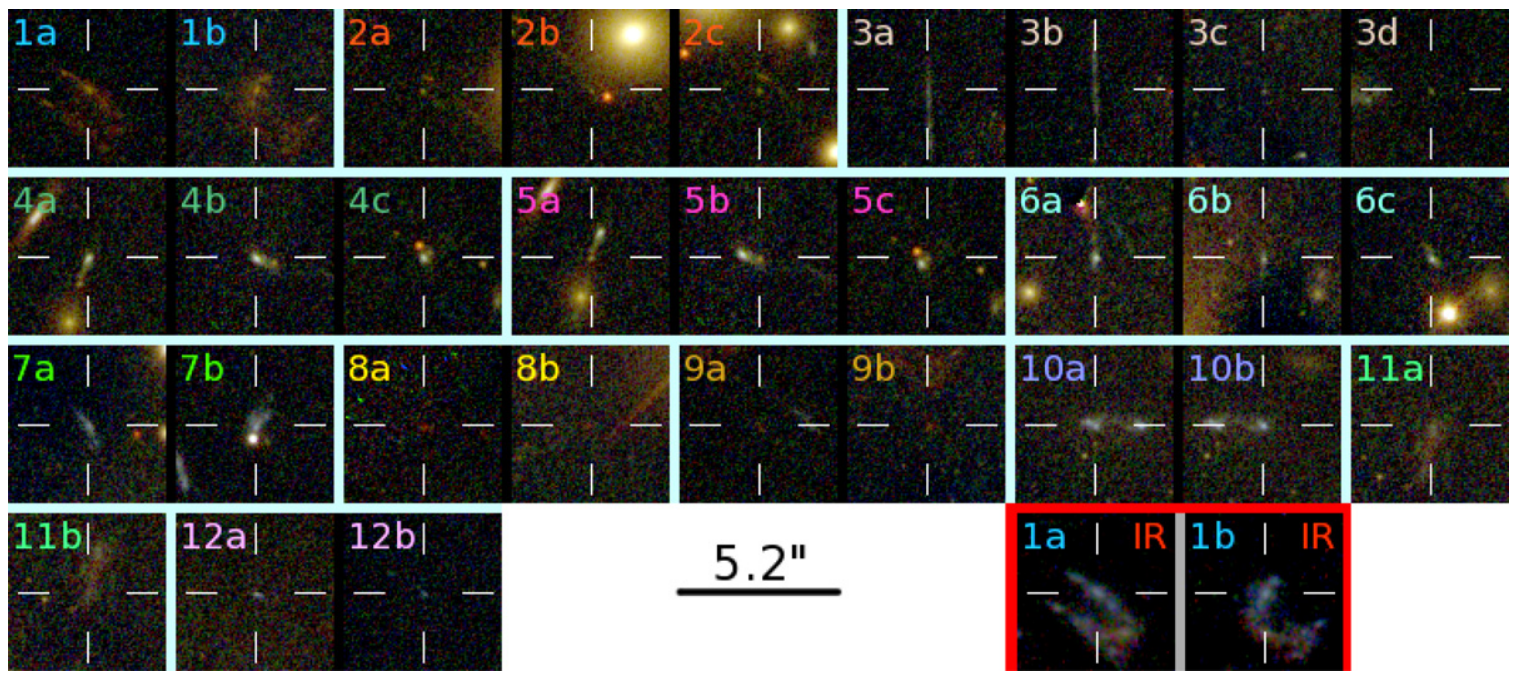

Figure 2. Multiple images of galaxies strongly lensed by A2261, as identified in this work (see Section 4.2, Figure 1 and Table 3 ). The BCG has been modeled and subtracted from all of these images. Most are ACS color images $(B=F 435 \mathrm{~W}+\mathrm{F} 475 \mathrm{~W}, \mathrm{G}=\mathrm{F} 606 \mathrm{~W}+\mathrm{F} 625 \mathrm{~W}$, and $\mathrm{R}=\mathrm{F} 775 \mathrm{~W}+\mathrm{F} 814 \mathrm{~W}+\mathrm{F} 850 \mathrm{LP})$, while those at bottom right use WFC3/IR filters $(\mathrm{B}=\mathrm{F} 105 \mathrm{~W}+\mathrm{F} 110 \mathrm{~W}, \mathrm{G}=\mathrm{F} 125 \mathrm{~W}+\mathrm{F} 140 \mathrm{~W}$, and $\mathrm{R}=\mathrm{F} 160 \mathrm{~W})$. Each image stamp is $5{ }^{\prime \prime} \cdot 2$ on a side.

(A color version of this figure is available in the online journal.)
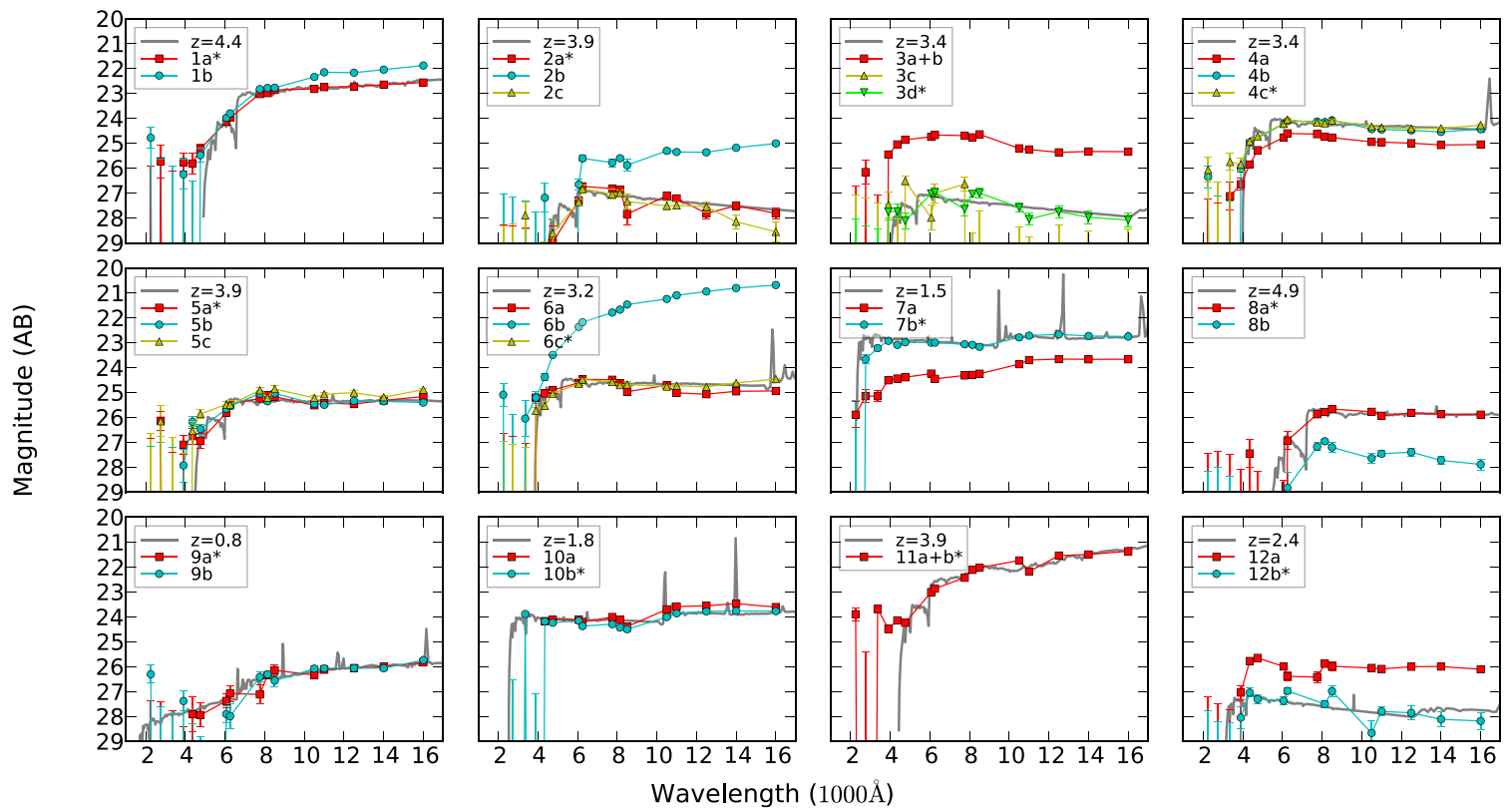

Wavelength $(1000 \AA)$

Figure 3. Spectral energy distributions (SEDs) of arcs as observed in our 16 HST filters. Also plotted in gray are BPZ SED fits to the best-isolated arcs marked with asterisks $(*)$ both in this figure and in Table 3. For each arc, the SEDs of all images should have similar shapes though these may shift vertically in magnitude as their magnifications may vary. The photometry of a few faint images may be contaminated by cluster light, despite our best efforts to model and subtract the BCG and other cluster galaxies. Most notably, $6 \mathrm{~b}$ is a faint image very near the BCG core.

(A color version of this figure is available in the online journal.)

$z \sim 1.5$, some of our models would predict a radial arc counterimage on the opposite side of the BCG core. We detect no such image. This search is aided by the fact that the arc is significantly detected in F390W, where most of the other arcs drop out and the BCG light is significantly reduced.

We initially identified a possible counterimage to this large arc with similar colors and photo- $z$ marked as "Y" in Figure 1 and colored magenta in Figure 4. However, the lens models required to reproduce this counterimage were significantly stronger (higher mass) than our final models described above and thus inconsistent with all of our multiple-image systems. They also predicted an additional multiple image to the north (in the vicinity of R.A., decl. [J2000] = 17:22:27.8, +32:08:16), which is not observed.

\subsection{Complementary Strong-lensing Analyses}

We performed semi-independent lens modeling analyses using Lenstool (Kneib 1993; Jullo et al. 2007) and LensPerfect (Coe et al. 2008, 2010). In the course of these analyses, we verified the multiple-image systems and estimated their redshifts independently.

Our Lenstool model consisted of an ellipsoidal NFW halo (Navarro et al. 1996) and truncated PIEMD (pseudo-isothermal elliptical mass distribution) halos (Kassiola \& Kovner 1993) 
Table 3

Multiple Images of Galaxies Strongly Lensed by A2261

\begin{tabular}{|c|c|c|c|c|c|}
\hline ID & $\begin{array}{c}\text { R.A. } \\
(\mathrm{J} 2000.0)\end{array}$ & $\begin{array}{c}\text { Decl. } \\
(\mathrm{J} 2000.0)\end{array}$ & $\begin{array}{c}\text { Magnitude } \\
\text { F775W (AB mag) }\end{array}$ & $\begin{array}{l}\text { Photometric } \\
\text { Redshift }^{a}\end{array}$ & $\begin{array}{l}\text { Lens Model } \\
\text { Redshift }^{b}\end{array}$ \\
\hline $1 \mathrm{a}$ & 172225.452 & +320825.02 & $23.02 \pm 0.04$ & $* 4.39_{-0.04}^{+0.03}$ & 4.44 \\
\hline$\underline{b}$ & 172227.295 & +320742.58 & $22.82 \pm 0.04$ & $0.48_{-0.03}^{+0.03}$ & $"$ \\
\hline $2 \mathrm{a}$ & 172226.338 & +320820.54 & $26.81 \pm 0.14$ & $* 3.89_{-0.13}^{+0.12}$ & 3.81 \\
\hline $\mathrm{b}$ & 172226.133 & +320818.59 & $25.78 \pm 0.16$ & $4.07_{-0.21}^{+0.11}$ & $"$ \\
\hline $\mathrm{c}$ & 172225.897 & +320817.16 & $27.04 \pm 0.20$ & $3.74_{-0.13}^{+0.14}$ & $"$ \\
\hline $3 a$ & 172228.917 & +320755.13 & $24.69 \pm 0.07$ & $0.26_{-0.01}^{+2.88}$ & 3.24 \\
\hline $\mathrm{b}$ & 172228.912 & +320753.96 & ${ }^{\prime}$ & $\dddot{n}^{-0.01}$ & $"$ \\
\hline $\mathrm{c}$ & 172226.972 & +320738.49 & $26.63 \pm 0.28$ & $2.60_{-0.77}^{+0.35}$ & $"$ \\
\hline d & 172225.391 & +320818.13 & $27.64 \pm 0.28$ & $* 3.38_{-0.21}^{+0.11}$ & $"$ \\
\hline $4 \mathrm{a}$ & 172228.569 & +320808.00 & $24.63 \pm 0.04$ & $3.48_{-0.03}^{+0.03}$ & 3.31 \\
\hline $\mathrm{b}$ & 172228.369 & +320737.51 & $24.12 \pm 0.04$ & $3.38_{-0.07}^{+0.07}$ & $"$ \\
\hline $\mathrm{c}$ & 172225.217 & +320813.52 & $24.17 \pm 0.05$ & $* 3.40_{-0.04}^{+0.04}$ & $"$ \\
\hline $5 \mathrm{a}$ & 172228.589 & +320807.15 & $25.23 \pm 0.09$ & $* 3.92_{-0.25}^{+0.17}$ & 3.31 \\
\hline $\mathrm{b}$ & 172228.323 & +320737.38 & $25.04 \pm 0.08$ & $0.29_{-0.04}^{+3.36}$ & $"$ \\
\hline $\mathrm{c}$ & 172225.202 & +320813.71 & $24.91 \pm 0.09$ & $3.35_{-2.85}^{+0.10}$ & $"$ \\
\hline $6 a$ & 172227.069 & +320809.30 & $24.49 \pm 0.07$ & $3.26_{-0.07}^{+0.01}$ & 3.09 \\
\hline $\mathrm{b}$ & 172227.115 & +320801.76 & $21.79 \pm 0.01$ & $0.22_{-0.03}^{+0.01}$ & $"$ \\
\hline $\mathrm{c}$ & 172228.615 & +320728.74 & $24.58 \pm 0.07$ & $* 3.24_{-0.03}^{+0.03}$ & $"$ \\
\hline $7 \mathrm{a}$ & 172226.599 & +320751.81 & $24.31 \pm 0.07$ & $1.57_{-0.02}^{+0.01}$ & 1.74 \\
\hline $\mathrm{b}$ & 172229.326 & +320759.35 & $23.06 \pm 0.05$ & $* 1.54_{-0.02}^{+0.01}$ & $"$ \\
\hline $8 a$ & 172226.158 & +320825.87 & $25.87 \pm 0.14$ & $* 4.92_{-0.13}^{+0.13}$ & 4.93 \\
\hline$\underline{b}$ & 172226.737 & +320741.61 & $27.17 \pm 0.17$ & $4.82_{-0.11}^{+0.05}$ & $"$ \\
\hline $9 a$ & 172225.304 & +320821.19 & $27.11 \pm 0.37$ & $* 0.78_{-0.19}^{+3.16}$ & 4.46 \\
\hline$\underline{b}$ & 172227.223 & +320739.20 & $26.41 \pm 0.19$ & $4.67_{-0.15}^{+0.13}$ & ” \\
\hline $10 \mathrm{a}$ & 172226.379 & +320801.89 & $24.01 \pm 0.05$ & $1.68_{-0.05}^{+0.04}$ & 1.67 \\
\hline$\underline{b}$ & 172226.261 & +320801.88 & $24.29 \pm 0.07$ & $* 1.79_{-0.23}^{+0.01}$ & $"$ \\
\hline $11 \mathrm{a}$ & 172226.854 & +320806.50 & $22.43 \pm 0.03$ & $* 3.88_{-0.04}^{+0.04}$ & 3.90 \\
\hline$\underline{b}$ & 172226.875 & +320805.01 & "c & ${ }^{\prime}$ & $"$ \\
\hline $12 \mathrm{a}$ & 172226.563 & +320812.81 & $26.42 \pm 0.21$ & $2.42_{-0.70}^{+0.05}$ & 2.83 \\
\hline $\mathrm{b}$ & 172229.045 & +320734.33 & $27.50 \pm 0.15^{d}$ & $* 2.43_{-0.26}^{+0.34}$ & $"$ \\
\hline
\end{tabular}

Notes.

${ }^{a}$ The best-isolated, least-contaminated arcs (marked with *) are chosen to provide the input redshift for each system. These are then optimized by the lens model. Note that robust photometric redshifts are not expected for arcs fainter than $\sim 26$ th magnitude. Uncertainties are formally $95 \%$ C.L. Broad, asymmetric error bars generally indicate a bimodal solution.

${ }^{\mathrm{b}}$ Results from LensPerfect.

${ }^{c}$ Continuous arcs were analyzed with a single photometric aperture for more robust photometric redshift estimates.

${ }^{\mathrm{d}}$ F814W.

for the 69 brightest cluster members, which were again identified photometrically but independently from the analysis in Section 4.1. We assumed core radii $r_{\text {core }}=300 \mathrm{pc}$ and scaling relations as in Jullo et al. (2007): velocity dispersion $\sigma_{0} \propto L^{1 / 4}$ and cutoff radius $r_{\text {cut }} \propto L^{1 / 2}$, giving all galaxies equal massto-light ratios. These two scaling normalizations are left as free parameters, adding to the six parameters contributed by the cluster halo: position $(x, y)$, ellipticity $(e, \theta)$, scale radius, and concentration.

For the LensPerfect analysis, we assumed a prior that the mass is densest near the center of the BCG and roughly decreases outward radially. Otherwise, it includes no assumptions about light tracing mass. Other priors include overall smoothness and rough azimuthal symmetry. For details, see Coe et al. (2008,
2010). The best solution found, according to these criteria, is shown in Figure 5. It perfectly reproduces the observed positions of all multiple images (to the accuracy with which they are input). We note that the solution is only well constrained within the white polygon, which bounds the multiple images and spans $13^{\prime \prime}<R<36^{\prime \prime}$.

\subsection{Results from the Ensemble of Models}

Our parametric mass models all yield acceptable fits $\left(\chi^{2}<\right.$ degrees of freedom) to the 30 observed image positions. The best Zitrin models reproduced these image positions with an rms of 0.92 and 1".02 for the spline and Gaussian smoothing, respectively. Assuming an intrinsic scatter of 1".4 (Section 4.1), these yield $\chi^{2}=12.9$ and 16.0 , respectively, with $29=(36-7)$ 


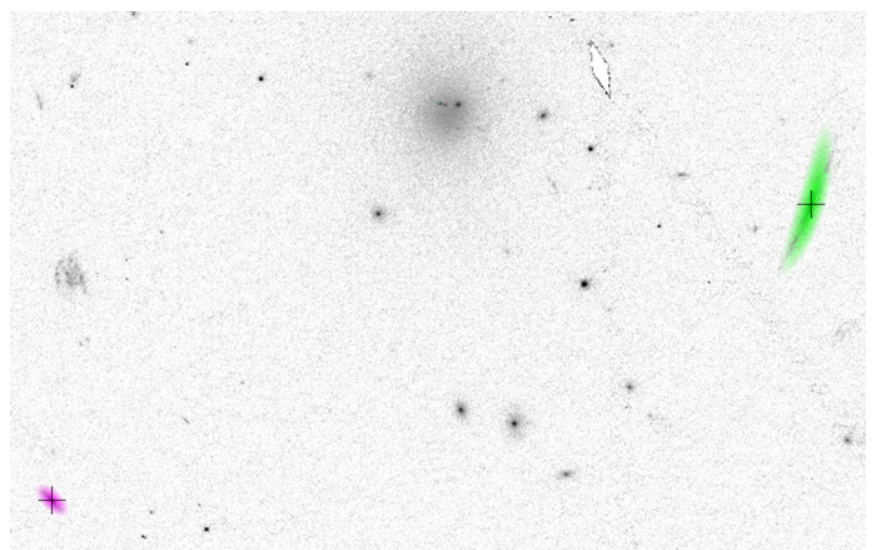

Figure 4. According to our strong-lens models constrained by 30 multiple images, the brightest arc $(z \sim 1.2)$ is strongly lensed and sheared but not multiply imaged. Shown in green is a circularly symmetric source lensed by one of our Zitrin (spline) lens models to the arc position as marked by the black cross. This is overlaid on the black and white UVIS F390W image. Another arc shown at bottom left has similar colors but is also consistent with being singly imaged, as shown in magenta. These arcs are also marked in Figure 1 as X and $\mathrm{Y}$, respectively.

(A color version of this figure is available in the online journal.)

degrees of freedom. The best Lenstool model, with an additional free parameter (eight total), reproduced the image positions with an rms of 0 '. 63 , yielding $\chi^{2}=6.13$ with 28 degrees of freedom. LensPerfect reproduces all observed image positions exactly as input.

Integrated projected mass profiles from our various SL mass models are presented in Figure 6. We adopt the average and scatter of these models as our primary SL constraints, but we also consider constraints from each method individually (Section 6). Some level of agreement is guaranteed by the fact that all models used the same input multiple images and photometric redshift information. These image identifications were verified independently in each analysis. The redshifts were allowed to vary somewhat and were optimized independently by each model.

The models converge most tightly on the projected mass contained within $\sim 20^{\prime \prime}$, roughly as expected given the Einstein radii of the systems. In Figure 7 , we plot the Einstein radius $R_{E}$ as a function of background source redshift $z_{s}$ for all four SL models. We calculated these $R_{E}\left(z_{s}\right)$ as those circular radii centered on the highest mass peak (coincident with the BCG) that enclose an average projected density equal to the critical SL density.

Our results, which range from $R_{E}=20^{\prime \prime} \pm 2^{\prime \prime}\left(z_{s}=1.5\right)$ to $22^{\prime \prime} \pm 0.5\left(z_{s}=2\right)$, are significantly lower than those roughly estimated from ground-based imaging as quoted by Umetsu et al. (2009): $R_{E}=40^{\prime \prime} \pm 4^{\prime \prime}\left(z_{s}=1.5\right)$. This previous estimate was based on $R_{E} \sim 30^{\prime \prime}$ for $z_{s} \lesssim 1$ assuming that the bright, prominent arc (marked with an $\mathrm{X}$ in Figure 5) lies near the Einstein radius. In this work, we find that this arc is not in fact located at $R_{E}$. Instead, it lies at $R \approx 27^{\prime \prime}$, greater than the $R_{E}=18^{\prime \prime} \pm 2^{\prime \prime}\left(z_{s}=1.2\right)$ determined robustly by our 12 other multiple-image systems. Furthermore, we find that it has a photo- $z \sim 1.2$, greater than that assumed in Umetsu et al. (2009). Both of these factors contributed to the higher concentration measured in that work.

\section{WEAK-LENSING MASS MODELING}

To probe the mass distribution of A2261 at larger radii, we turn to WL analyses of wider ground-based images obtained with Subaru Suprime-Cam, as previously studied in detail by Umetsu et al. (2009) and Okabe et al. (2010). Here we present new, more robust analyses incorporating additional observations, improved image reductions, and new analytical techniques. The additional observations include KPNO Mayall $4 \mathrm{~m}$ imaging (Section 5.1) and spectroscopy from MMT/Hectospec (Section 3).

\subsection{Subaru and KPNO Wide-field Imaging}

Our WL analysis is based on archival $B_{\mathrm{J}} V_{\mathrm{J}} R_{\mathrm{C}}$ imaging obtained with the $34^{\prime} \times 27^{\prime}$ FOV Suprime-Cam (Miyazaki et al. 2002) on the Subaru $8.2 \mathrm{~m}$ telescope and NOAO archival MOSAIC1 $i^{\prime} z^{\prime}$ imaging obtained with the Mayall $4 \mathrm{~m}$ telescope at KPNO (program 2008A-0356, PI. Mandelbaum). The integration times are 20,30, 45, 60, and 90 minutes, respectively, for the five filters $B_{\mathrm{J}} V_{\mathrm{J}} R_{\mathrm{C}} i^{\prime} z^{\prime}$. The Subaru $R_{\mathrm{C}}$-band images used for galaxy shape analyses have a seeing FWHM $\approx$ $00^{\prime \prime} 65$ and were obtained at two different orientations rotated by $90^{\circ}$ (see Section 5.4).

\subsection{Previous Analyses}

Umetsu et al. (2009) and Okabe et al. (2010) both measured WL distortions in the Subaru $R_{\mathrm{C}}$-band imaging described above. Background galaxies were selected based on $V_{\mathrm{J}} R_{\mathrm{C}}$ color-magnitude cuts determined in part so as to minimize
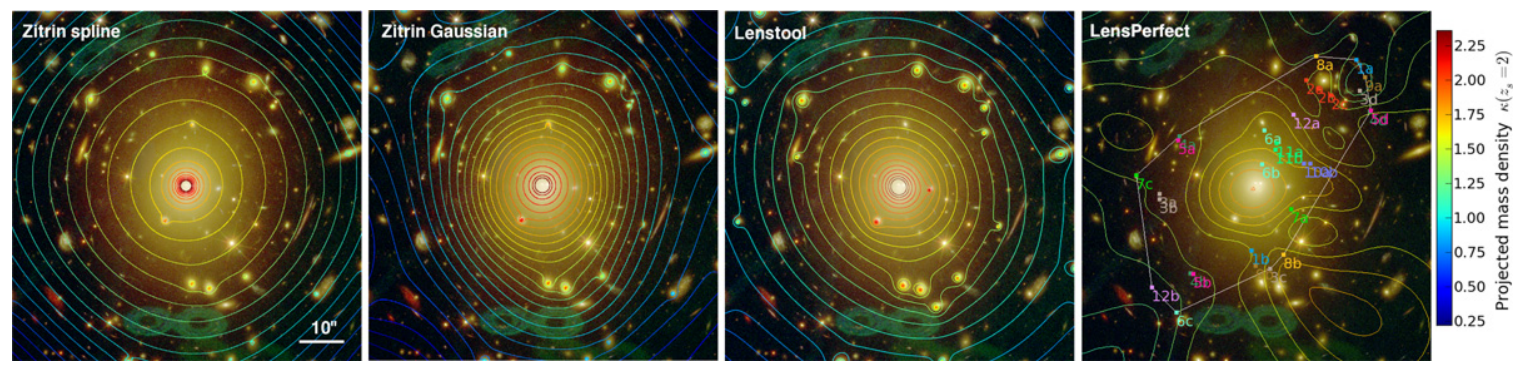

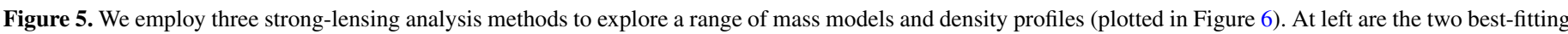

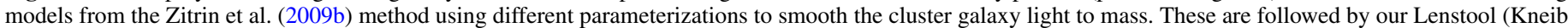

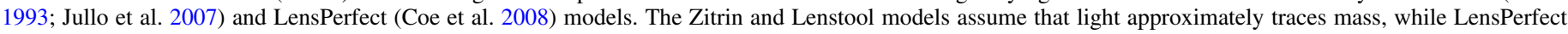

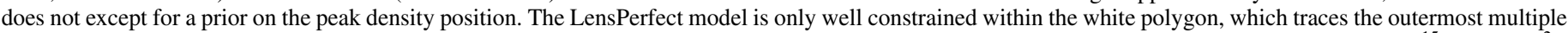

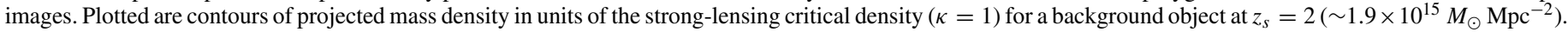

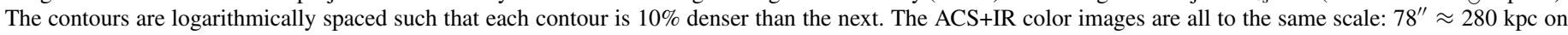
a side.

(A color version of this figure is available in the online journal.) 


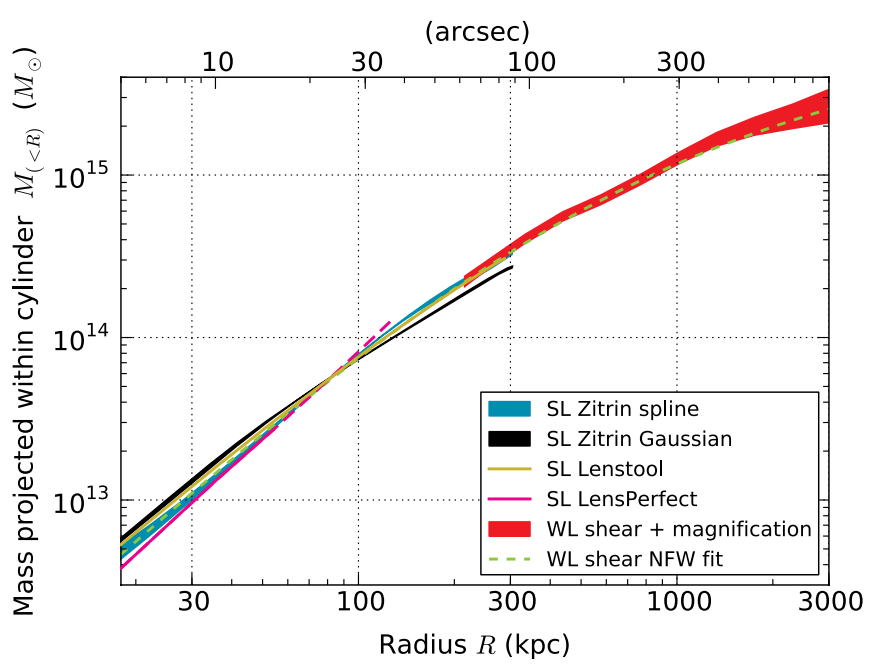

Figure 6. Projected two-dimensional mass profiles from strong and weak lensing. We present four SL models and two WL models. Two of the SL models are based on the Zitrin method (Section 4.1) using different kernels to smooth the light distribution for use as the halo mass model. The LensPerfect model is steepest in integrated $M(<R)$, which translates to the shallowest density profile $\kappa(R)$, while the Lenstool model is about average (Section 4.3).

(A color version of this figure is available in the online journal.)

contamination of unlensed cluster galaxies as described in Medezinski et al. (2007) and Umetsu \& Broadhurst (2008).

Both analyses identified a massive background structure at $z \sim 0.5$ that may affect the lensing signal. Umetsu et al. (2009) found that out of four clusters analyzed, A2261 was the most sensitive to the exact profile fitting method used.

The first method, used in both papers (and commonly elsewhere), fits the observed shears (binned radially) directly to those expected from NFW profiles. The second method attempts to correct for the mass-sheet degeneracy based on the observed shears alone. With the outer "mass sheet" density left as a free parameter $\kappa_{b}$, the discretized density profile is iteratively refined toward consistency with the observed reduced tangential shears $g_{+}=\gamma_{+} /(1-\kappa)$. This method (Umetsu \& Broadhurst 2008; Umetsu et al. 2009) is a nonlinear extension of earlier "aperture densitometry" techniques developed by Fahlman et al. (1994) and Clowe et al. (2000).

Umetsu et al. (2009) found that NFW fits to the profile derived from the latter method yield a marginally higher mass concentration $c_{\mathrm{vir}}=10.2_{-3.5}^{+7.1}$ than the $c_{\mathrm{vir}}=6.4_{-1.4}^{+1.9}$ obtained using the former method (Table 4 and Figure 10). This higher value was supported by their rough estimate of the SL Einstein radius $R_{E} \sim 40^{\prime \prime}\left(z_{s} \sim 1.5\right)$ based on the prominent arc (see Section 4.4). A joint fit to the WL and $R_{E}=(40 \pm 4)^{\prime \prime}$ yielded $c_{\text {vir }}=11.1_{-1.9}^{+2.2}$, which was quoted by Oguri et al. (2009) as an example of a cluster with higher-than-expected concentration.

We revisit the Umetsu et al. (2009) $c_{\text {vir }} \sim 10 \mathrm{WL}$ result as follows. We have calculated an additional constraint $\bar{\kappa}\left(<1^{\prime}\right)$ corresponding to the mean density interior to the inner radial boundary of WL measurements. Based on its inclusion, the bestfit value and uncertainties both decrease from $c_{\text {vir }}=10.2_{-3.5}^{+7.1}$ to $c_{\text {vir }}=5.8_{-1.4}^{+1.8}$. The results are consistent within their uncertainties, but the precision is significantly improved by increasing the radial range of constraints. Previous analyses have often neglected to calculate and include this innermost data point (e.g., Umetsu et al. 2009; Oguri et al. 2009).

Okabe et al. (2010) found a lower virial mass $M_{\text {vir }}=$ $1.36_{-0.24}^{+0.28} \times 10^{15} M_{\odot}$ and $c_{\mathrm{vir}}=6.04_{-1.31}^{+1.71}$. This profile underesti-

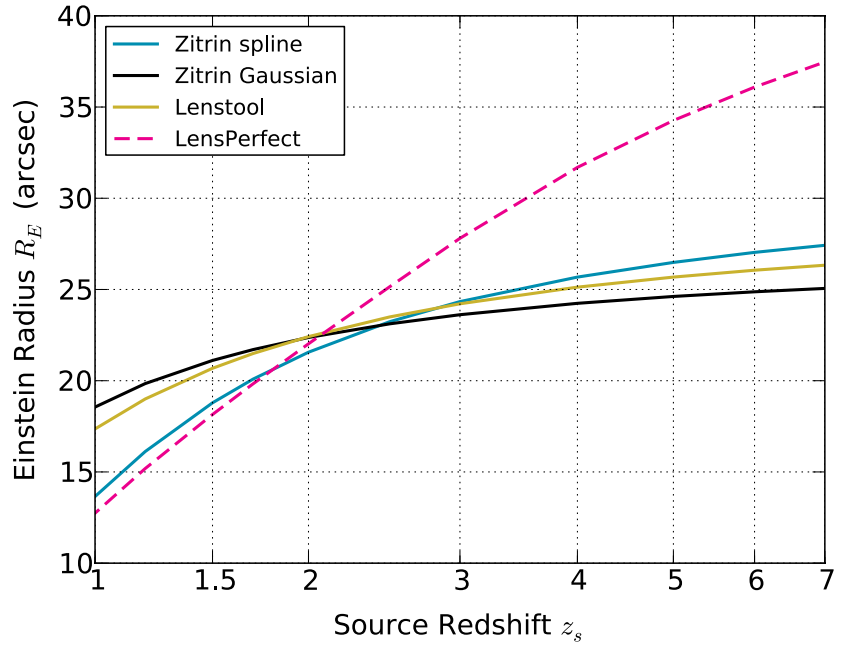

Figure 7. Einstein radius $R_{E}$ as a function of source redshift $z_{s}$ measured by our various lens models (with mass profiles plotted in Figure 6). Our results are much lower than the rough estimate of $R_{E} \sim 40^{\prime \prime}\left(z_{s}=1.5\right)$ from ground-based imaging quoted by Umetsu et al. (2009). Instead, our models range between $R_{E}=20^{\prime \prime} \pm 2^{\prime \prime}\left(z_{s}=1.5\right)$ and $22^{\prime \prime} \pm 0.5\left(z_{s}=2\right)$. Some level of agreement is guaranteed by the fact that all models used the same input multiple images and photometric redshift information, though the redshifts are allowed to vary somewhat and are optimized independently by each method. The LensPerfect mass profile is shallow, hovering near the lensing critical density, allowing unconstrained perturbations beyond $R \gtrsim 30^{\prime \prime}$ (including some area outside the multiple-image constraints) to significantly influence $R_{E}$ at these radii.

(A color version of this figure is available in the online journal.)

mates mass in our SL region by $\sim 20 \%$ (Section 6). We compared our shear catalog directly with that used in Okabe et al. (2010) and provided to us. We find similar WL signal for $R>3^{\prime}$ but recover stronger signal interior to this radius. This difference is most likely explained by improved background selection in our catalog with lower contamination due to cluster galaxies (Section 5.5).

\subsection{Current Analysis}

In addition to the Subaru $V_{\mathrm{J}}$ and $R_{\mathrm{C}}$ images, we also utilize the $B_{\mathrm{J}}$-band image, which improves our selection of background galaxies (Section 5.5) with respect to the previous analyses. Our Subaru image reduction procedure (Nonino et al. 2009) is somewhat improved compared to that used in Umetsu et al. (2009) in terms of distortion corrections and image co-addition (here point-spread function (PSF) weighted). After trimming the shallower edges, the final co-added images roughly cover a circular area with a 17!.8 radius $\left(\sim 1000 \operatorname{arcmin}^{2}\right)$, which we use for our analysis. As in the previous analyses, we measured galaxy shapes in the Subaru $R_{\mathrm{C}}$-band images, though our procedure is slightly different (Section 5.4).

The KPNO $i^{\prime}$ and $z^{\prime}$ images were reduced using calibration frames, including fringe and pupil maps, obtained from the archive, and then stacked in a manner similar to the Subaru images. Zero points were calibrated based on comparisons with point-source photometry from SDSS DR7 (Abazajian et al. 2009).

Five-band $B_{\mathrm{J}} V_{\mathrm{J}} R_{\mathrm{C}} i^{\prime} z^{\prime}$ photometry was measured using SExtractor in PSF-matched images created by ColorPro (Coe et al. 2006). Subaru zero points were calibrated based on comparisons to $H S T$ and KPNO photometry and then recalibrated based on SED fits to photometry of galaxies with measured spectroscopic redshifts primarily from Hectospec and supplemented by SDSS DR7 (Section 3). 
Table 4

Published NFW Fits to the Mass Profile of A2261

\begin{tabular}{|c|c|c|c|}
\hline $\begin{array}{l}\text { Virial Mass } \\
M_{\text {vir }}\left(10^{15} M_{\odot} h_{70}^{-1}\right)\end{array}$ & $\begin{array}{c}\text { Concentration } \\
c_{\mathrm{vir}}\end{array}$ & Article & Constraints \\
\hline $1.93_{-0.31}^{+0.37}$ & $6.4_{-1.4}^{+1.9}$ & Umetsu et al. (2009) & 1) WL shears \\
\hline $1.80_{-0.24}^{+0.29}$ & $10.2_{-3.5}^{+7.1}$ & Umetsu et al. (2009) & 2) WL shears + estimated mass sheet ${ }^{a}$ \\
\hline $1.79_{-0.23}^{+0.24}$ & $11.1_{-1.9}^{+2.2}$ & Umetsu et al. (2009) & 3) $\mathrm{WL}(2)+$ Einstein radius estimate: $^{b} R_{E}\left(z_{s}=1.5\right)=(40 \pm 2)^{\prime \prime}$ \\
\hline $1.36_{-0.24}^{+0.29}$ & $6.0_{-1.3}^{+1.7}$ & Okabe et al. (2010) & 4) WL shears \\
\hline $1.89_{-0.34}^{+0.41}$ & $5.8_{-1.4}^{+1.8}$ & This work & 5) $\mathrm{WL}(2)$ re-analyzed ${ }^{c}$ \\
\hline $2.09_{-0.27}^{+0.31}$ & $6.0_{-0.9}^{+1.1}$ & This work & 6) WL shears \\
\hline $1.89_{-0.22}^{+0.25}$ & $6.7_{-1.0}^{+1.1}$ & This work & 7) WL shears + estimated mass sheet ${ }^{d}$ \\
\hline $2.21_{-0.23}^{+0.25}$ & $5.7_{-0.7}^{+0.8}$ & This work & 8) WL shears + magnification (number count depletion) \\
\hline $1.97_{-0.21}^{+0.26}$ & $6.6_{-0.4}^{+0.5}$ & This work & 9) $\mathrm{SL}+\mathrm{WL}(5)$ \\
\hline $1.98_{-0.16}^{+0.19}$ & $6.6_{-0.4}^{+0.4}$ & This work & 10) $\mathrm{SL}+\mathrm{WL}(7)$ \\
\hline $2.21_{-0.15}^{+0.21}$ & $6.2_{-0.3}^{+0.3}$ & This work & 11) $\mathrm{SL}+\mathrm{WL}(8)=$ our primary result when assuming a spherical halo \\
\hline $1.70_{-0.12}^{+0.16}$ & $4.6_{-0.2}^{+0.2}$ & This work & 12) $\mathrm{SL}+\mathrm{WL}(8)+\mathrm{X}$-ray, with one model for halo elongation \\
\hline
\end{tabular}

Notes. We also roughly identify and estimate the effects of background/foreground structures along the line of sight (Section 6.1). We find that correcting for these may lower $M_{\mathrm{vir}}$ by $\sim 7 \%$ and increase $c_{\mathrm{vir}}$ by $\sim 5 \%$.

a The mass sheet density $\kappa_{b}$ in the outer annulus was estimated based on an NFW fit to the shears.

b In this work, we find a lower $R_{E}\left(z_{s}=1.5\right)=(20 \pm 2)^{\prime \prime}$.

${ }^{c}$ Now including the innermost bin $\bar{\kappa}\left(<1^{\prime}\right)$, the mean convergence interior to the weak-lensing measurements.

${ }^{\mathrm{d}}$ Iterative NFW fitting is performed to find the best-fitting mass sheet density $\kappa_{b}$.

\subsection{Shape Measurement}

We produced two separate co-added $R_{\mathrm{C}}$-band images for the shape analyses based on the imaging obtained at two different orientations separated by $90^{\circ}$. Galaxy shapes (reduced shears) were measured at each orientation and their weighted averages computed. In both Umetsu et al. (2009) and Okabe et al. (2010), shapes were instead measured in co-added images that combined both orientations. We find that this does not have a significant effect on the derived mass profile. When we use the same method to analyze shears measured in Umetsu et al. (2009) and in this work, we find consistent results (Table 4, Rows 5 and 6).

For accurate shape measurements of faint background galaxies, we used the IMCAT software (Kaiser et al. 1995), following the formalism outlined in that paper. Full details of our WL analysis pipeline are provided in Umetsu et al. (2010). We have tested our shape measurement and object selection pipeline using simulated Subaru Suprime-Cam images (M. Oguri 2010, private communication; Massey et al. 2007). We recover input WL signals with good precision: typically a shear calibration bias $|m| \lesssim 5 \%$ (where this bias shows a modest dependence of calibration accuracy on seeing conditions) and a residual shear offset $c \sim 10^{-3}$, which is about one order of magnitude smaller than the typical distortion signal (reduced shear $|g| \sim 10^{-2}$ ) in cluster outskirts. This level of performance is comparable to other similarly well tested methods (Heymans et al. 2006) and has been improved in comparison with our previous pipeline used in Umetsu et al. (2009), which achieved 5\% $\lesssim|m| \lesssim 10 \%$.

\subsection{Background Galaxy Selection}

Robust selection of background galaxies is crucial in WL analyses to minimize contamination by unlensed cluster and/or foreground galaxies that would dilute the lensing signal by a fraction equal to the level of contamination (Broadhurst et al. 2005b; Medezinski et al. 2007). If not accounted for properly, contamination can be especially significant at small clustercentric radii, where cluster galaxies are relatively dense. Previous analyses have demonstrated that color-color selection using three Subaru broadband filters delivers robust discrimination between cluster, foreground, and background galaxies (Medezinski et al. 2010, 2011; Umetsu et al. 2010, 2011a, 2011b).

Here we began by detecting objects within 17 '.8 ( 3.8 Mpc) of the BCG (the area deeply imaged by Subaru). We pruned stars from this sample based on $R_{\mathrm{C}}$-band magnitude, peak flux, FWHM, and SExtractor "stellarity."

We then derived $B_{\mathrm{J}} V_{\mathrm{J}} R_{\mathrm{C}}$ color-color-magnitude cuts (Figure 8) as described in Medezinski et al. (2007, 2010, 2011). We calculated number count density and average clustercentric radius both as a function of position in this color-color space. Cluster galaxies are identified as a peak in the former and minimum in the latter. We determined the region occupied by these galaxies and later found it to coincide well with colors of cluster members as determined based on a velocity caustic analysis of Hectospec spectroscopic redshifts (Section 3).

We then defined regions in this color-color space well separated from the cluster galaxies for use as our background galaxy selection. The border placement is optimized to maximize total number counts while minimizing contamination from cluster members. The latter can be detected as dilution of the average shear signal and/or a rise in number counts toward the cluster center. We also imposed magnitude cuts $22<R_{\mathrm{C}}<26$ to further avoid contamination at the bright end and incompleteness at the faint end, while maximizing the number of faint galaxies that contribute to the lensing signal.

Our final cuts (Figure 8) yielded 12,762 background galaxies (12.8 $\left.\operatorname{arcmin}^{-2}\right)$ for WL analysis. We verified that the WL shear signal increases toward the center and that the B-mode (curl component) is consistent with zero. We later verified that these cuts successfully reject all 189 galaxies identified spectroscopically as cluster members within the Subaru FOV (Section 3).

To estimate the mean effective redshift of this background population, we applied this same color-color-magnitude cut to galaxies with robust photometry and photometric redshifts measured in the COSMOS field (Capak et al. 2007; Ilbert et al. 


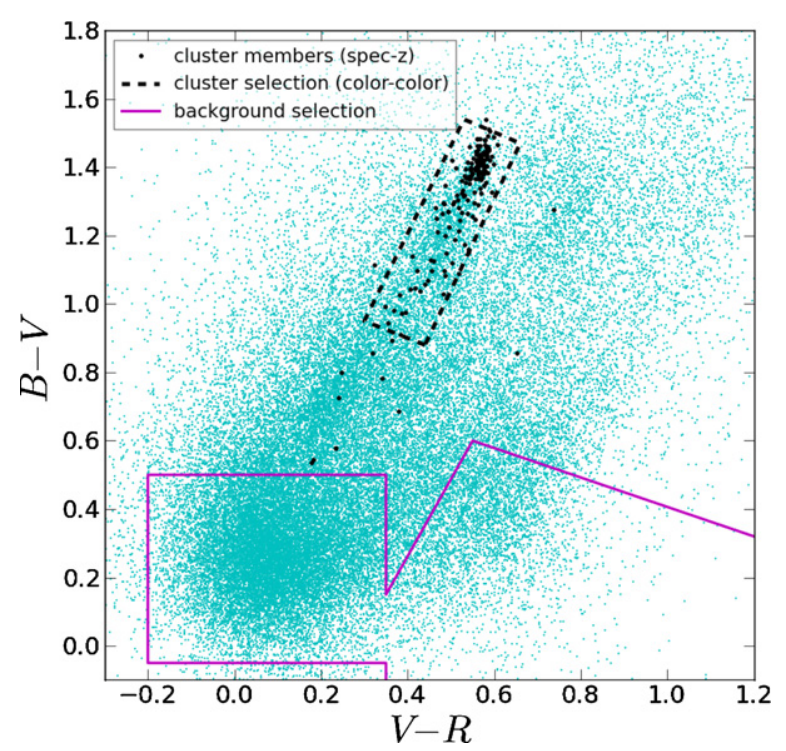

Figure 8. Background galaxies are selected for weak-lensing analysis (lower outlined region) based on Subaru $B_{\mathrm{J}} V_{\mathrm{J}} R_{\mathrm{C}}$ color-color-magnitude selection. At small radius, the cluster overdensity is identified as the dashed region. The background samples are well isolated from this region and satisfy other criteria as discussed in Section 5.5. This background selection successfully excludes all 189 cluster members (black) within the Subaru FOV as identified based on our velocity caustic analysis of Hectospec spectroscopic redshifts (Section 3). Cyan points are $R_{\mathrm{C}}<26$ galaxies, where stars have been identified and excluded.

(A color version of this figure is available in the online journal.)

2009). We compute the average lensing efficiency $\beta=D_{L S} / D_{S}$ for this sample given our lens redshift $z_{L}=0.225$ and find an effective $z_{S}=0.99 \pm 0.10$ for our background WL sample. For each lensed galaxy, the factor $\beta$ is a function of angular diameter distances from lens to source $D_{L S}=D_{A}\left(z_{L}, z_{S}\right)$ and observer to source $D_{S}=D_{A}\left(0, z_{S}\right)$. We later marginalized over this uncertainty when fitting mass profiles to our WL data.

\subsection{Revised Weak-lensing Mass Profile}

In addition to our revised selection of background galaxies, we also used a slightly different method to estimate the "masssheet," or background density $\kappa_{b}$. Here, we performed iterative NFW fitting, allowing $\kappa_{b}$ to be a free parameter (Umetsu et al. 2010).

We then performed a second analysis method that incorporates independent WL magnification data (depleted number counts of faint background galaxies) in a Bayesian approach. For details on this method, see Umetsu et al. (2011a, 2011b). Measurements of number count depletion generally break the mass sheet degeneracy more robustly (e.g., Broadhurst et al. 1995; Umetsu et al. 2011b) than the aperture densitometry technique described above.

We find a consistent mass profile solution based on a joint Bayesian fit to both the observed shears and magnification as shown in Figure 9. The total signal-to-noise ratio $(\mathrm{S} / \mathrm{N})$ in our tangential distortion profile is $\mathrm{S} / \mathrm{N} \approx 17$ (defined as in Equation (9) of Okabe et al. 2010), whereas $S / N \approx 20$ in the joint mass profile from combined tangential reduced shear and magnification measurements (Equation (38) of Umetsu \& Broadhurst 2008). Thus, in addition to breaking the mass sheet degeneracy, the magnification measurements also increased the overall significance by $\sim 20 \%$ (cf. Table 5 of Umetsu et al. 2011a; Rozo \& Schmidt 2010).

Our $B_{\mathrm{J}} V_{\mathrm{J}} R_{\mathrm{C}}$ color-color selection does not allow us to effectively discriminate between "blue" and "red" background samples with properties similar to those derived from $B_{\mathrm{J}} R_{\mathrm{C}} z^{\prime}$ color-color selection (Medezinski et al. 2010, 2011). Galaxies in the "blue" samples identified in these works have steep number count slopes, roughly canceling out any number count depletion.

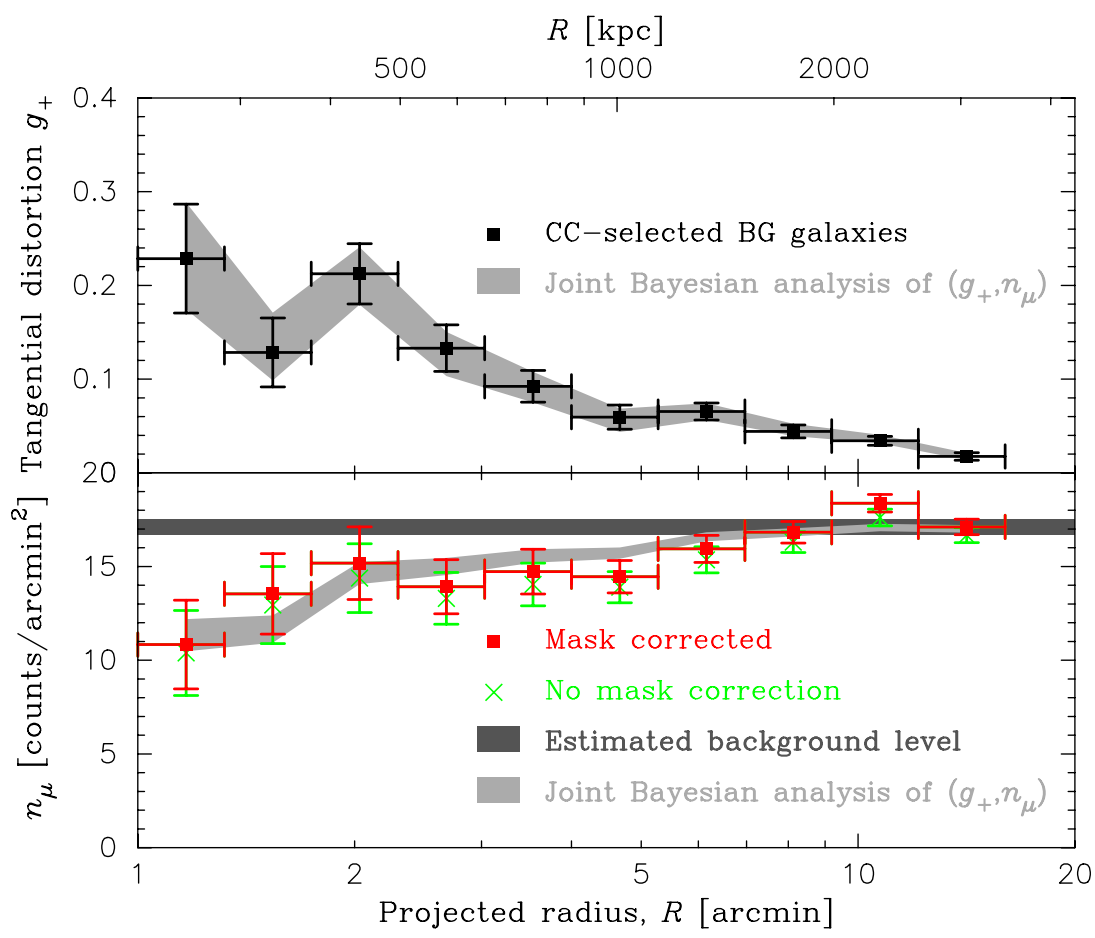

Figure 9. Weak-lensing tangential reduced shear (top) and magnification (number count depletion) profiles (bottom) measured in Subaru images of A2261 (Section 5.6). Also plotted is a joint Bayesian fit to both. This is our primary WL model. Bright objects are masked out to refine the estimates of area and thus number count densities. (A color version of this figure is available in the online journal.) 


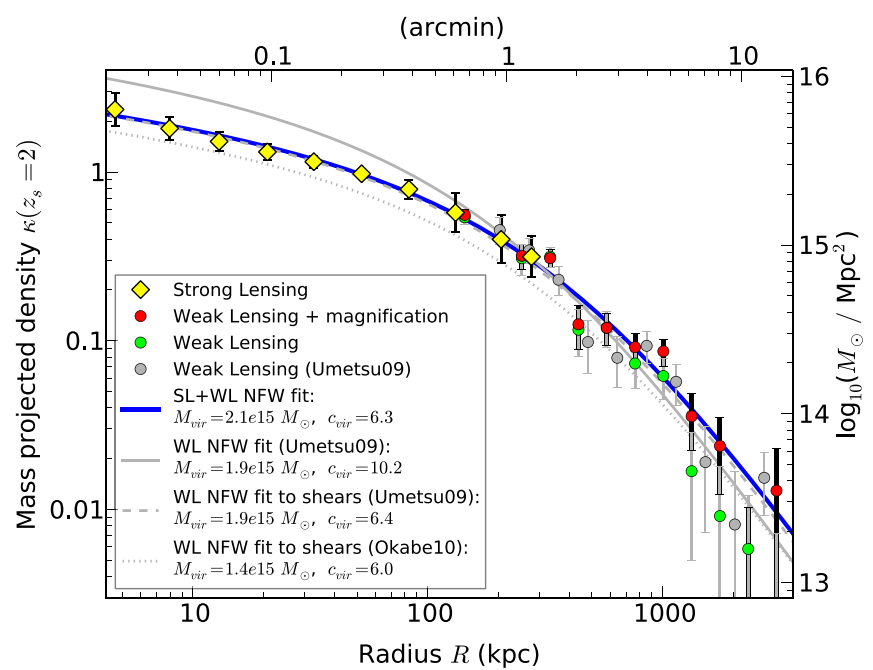

Figure 10. Projected mass density profile derived from strong- and weak-lensing analyses fit to NFW profiles from published analyses (gray) and this work (colors). The SL results are the average and scatter of our various mass models (Section 4.4).

(A color version of this figure is available in the online journal.)

Stronger magnification signals are measured in "red" $B_{\mathrm{J}} R_{\mathrm{C}} z^{\prime}$ samples with relatively flatter number counts.

To investigate the effect this may have on our analysis, we explored the $B_{\mathrm{J}} R_{\mathrm{C}} z^{\prime}$ colors of the subset of our galaxies detected in shallower $z^{\prime}$-band KPNO imaging. We found that the majority of our background sample corresponds to a "red" selection in $B_{\mathrm{J}} R_{\mathrm{C}} z^{\prime}$, as desired. We repeated our magnification analysis on this red subset and found no significant changes in our results except for somewhat larger uncertainties due to the lower number of galaxies.

\section{MASS PROFILE FROM JOINT STRONG- + WEAK-LENSING ANALYSIS}

For a mass concentration determination that is both precise and accurate, the inner mass profile must be simultaneously constrained by SL and WL analyses (e.g., Meneghetti et al. 2010). In Section 4, we derived a range of SL mass profiles from which we calculated the average with uncertainties. Then in Section 5 we presented various WL analyses. Our final WL analysis including both shear and magnification (number count depletion) information is our most robust. The magnification data break the mass sheet degeneracy and increase our overall WL S/N.

Our SL and WL data agree well in their region of overlap (Figure 10). We perform joint NFW fitting to the SL projected mass enclosed $M(<R)$ measured at 12 points $5^{\prime \prime} \leqslant R \leqslant 1^{\prime}$ $(18-215 \mathrm{kpc})$ and the WL projected mass density $\kappa(R)$ measured in 11 bins with centers $40^{\prime \prime} \leqslant R \lesssim 14^{\prime} .2$ (144-3059 kpc). We note that SL arcs fundamentally constrain $M(<R)$, not $\kappa(R)$, and that the former yields tighter constraints on the profile concentration (Coe et al. 2010, their Figure 9). In the case of a single Einstein ring, for example, the projected density profile $\kappa(R)$ is uncertain and strongly correlated from one radial bin to the next as it must add to the correct mass enclosed within the Einstein radius $M\left(<R_{E}\right)$. The WL observables, on the other hand, more fundamentally constrain $\kappa(R)$, which enters into the equations for shear and magnification (e.g., Kaiser 1995; Broadhurst et al. 1995; Umetsu et al. 2011a).

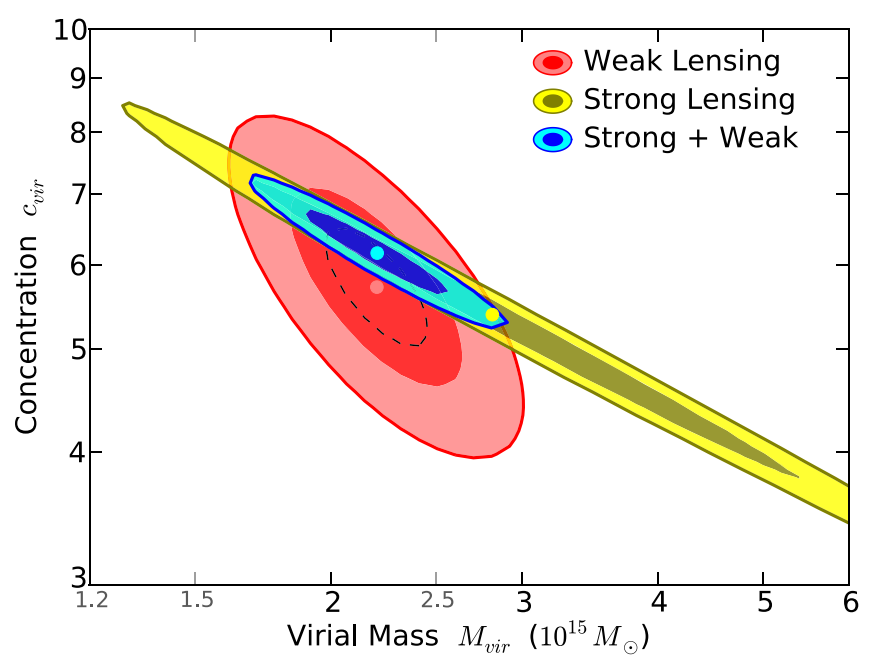

Figure 11. Constraints on the virial mass and concentration of A2261 from lensing analyses. Contours are $1 \sigma$ and $2 \sigma\left(\Delta \chi^{2}=2.30\right.$ and 6.17 , or $\approx 68 \%$ and $95 \%$ confidence assuming Gaussian ellipsoidal uncertainties). The dashed black line is $1 \sigma$ for weak lensing if marginalizing over one of the variables $\left(\Delta \chi^{2}=1\right)$. Best-fit values are also indicated. We marginalized over the weaklensing background redshift uncertainty $z_{s}=0.99 \pm 0.10$.

(A color version of this figure is available in the online journal.)

Our joint SL+WL fitting yields a virial mass $M_{\text {vir }}=(2.2 \pm$ $0.2) \times 10^{15} M_{\odot} h_{70}^{-1}$ and concentration $c_{\text {vir }}=6.2 \pm 0.3$ with a significantly greater precision than that obtained by WL alone $\left(c_{\text {vir }}=5.7_{-0.7}^{+1.0}\right)$. Confidence contours are plotted in Figure 11, and the constraints are tabulated in Table 4. Our new results strongly disfavor the previous $c_{\text {vir }} \sim 10$ results (Umetsu et al. 2009).

Our averaged SL constraints include two MCMC chains from the Zitrin method plus one model each from Lenstool and LensPerfect. We verify that this choice does not significantly affect our results. If we consider the Lenstool model only (assuming the uncertainties derived from the scatter among all models), we find that the joint SL+WL constraints yield $M_{\text {vir }}$ lower by $\sim 5 \%$ and $c_{\text {vir }}$ lower by $\sim 0.04 \%$. Similarly for the flatter LensPerfect mass profile within $36^{\prime \prime}$, we find $M_{\text {vir }}$ higher by $\sim 5 \%$ and $c_{\text {vir }}$ lower by $\sim 3 \%$. While the best fits do not change significantly, the advantage of using all models is to robustly estimate the uncertainties.

Our use of the spherical NFW profile enables the most direct comparisons with analyses of simulated halos fit exclusively to this profile (e.g., Klypin et al. 2011; Prada et al. 2012; Bhattacharya et al. 2011). Other mass profiles, including the Einasto (1965) profile, have been shown to yield slightly better fits to simulated halos (Navarro et al. 2004; Merritt et al. 2005, 2006; Navarro et al. 2010). The choice of profile does not significantly affect the derived concentrations (Duffy et al. 2008; Gao et al. 2008; Reed et al. 2011).

\subsection{Effect of Background Structures}

LOS structures introduce noise into measurements of both SL (Jullo et al. 2010; D’Aloisio \& Natarajan 2012; Host 2012) and WL (Hoekstra et al. 2011; Becker \& Kravtsov 2011; Gruen et al. 2011; Bahé et al. 2012). Strongly lensed arcs are observed at higher redshifts on average than weakly lensed galaxies, so the amplitude of the SL cosmic noise is higher. However, for a massive cluster, the relative significance is higher in the outskirts probed by WL where the cluster density drops off (see, e.g., Figure 1 of Umetsu et al. 2011a). Thus, it is most important to 

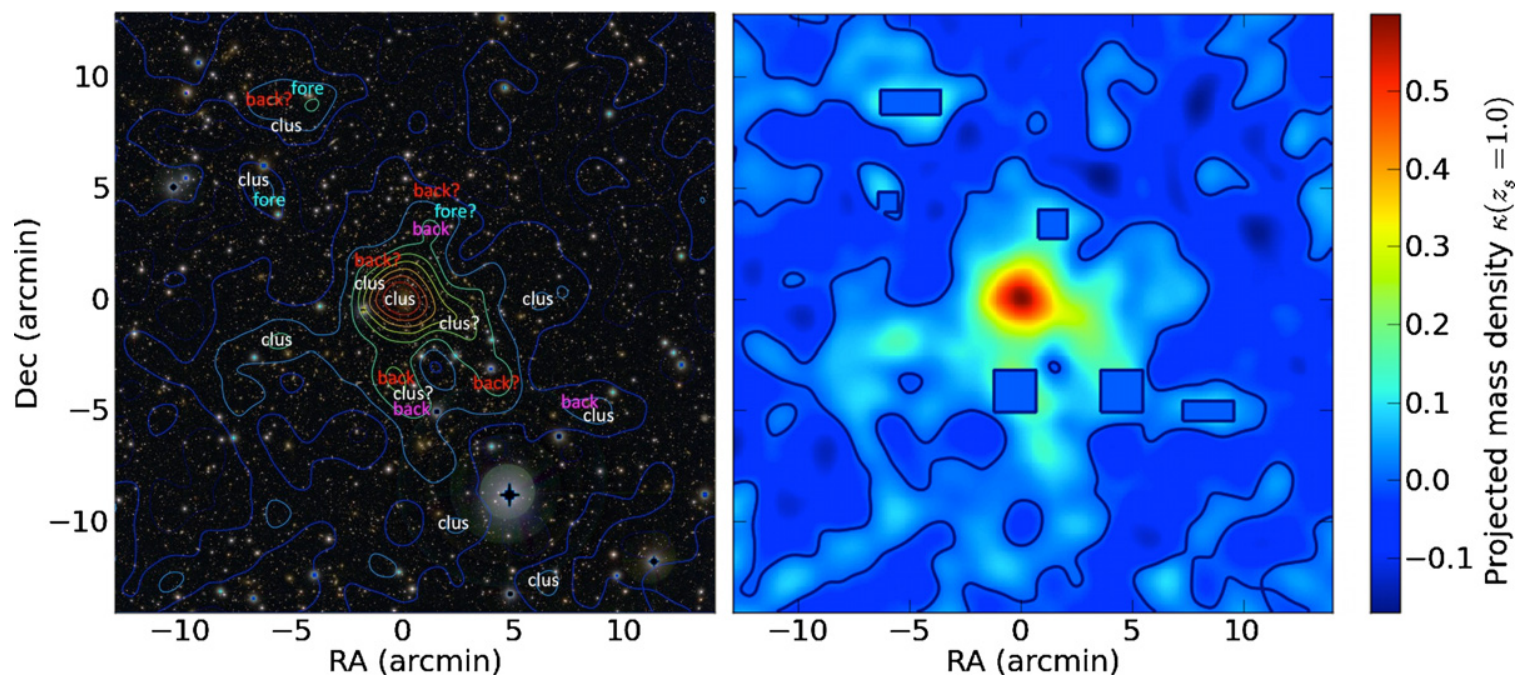

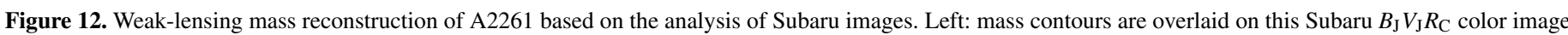

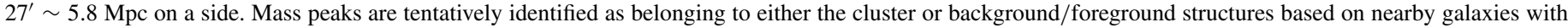

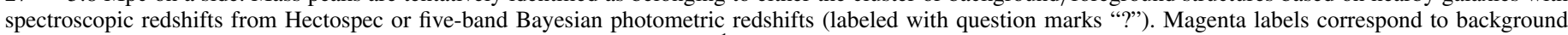

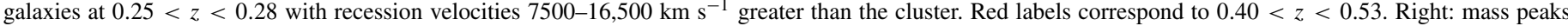

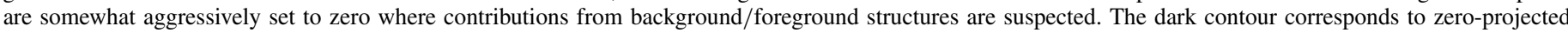

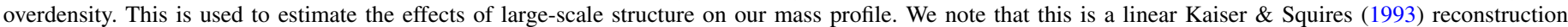
smoothed with a Gaussian kernel unlike the analysis discussed in Section 5 but necessary to yield a mass map suitable for this estimate.

(A color version of this figure is available in the online journal.)

include these uncertainties in the WL analysis and correct for the effects if possible.

We added a cosmic covariance matrix to the WL measurements as in Umetsu et al. (2012) and found that the best-fit mass and concentration are robust at the few percent level, and the statistical uncertainties are similar to those from the shape measurements. Rather than simply adding this statistical noise to our uncertainties, here we estimate a correction for the WL effects of large-scale structure as observed specifically behind A2261 based on the available data. We then propagate this correction to our joint SL+WL constraints.

Significant structure behind A2261 was identified by Umetsu et al. (2009) and Okabe et al. (2010) and estimated to be at $z \sim 0.5$ based on $V_{\mathrm{J}}-R_{\mathrm{C}}$ galaxy colors in that region. It was posited that this structure could bias the derived WL signals. Here we present a rough estimate of the effects of background structures on our derived mass and concentration.

We identified mass peaks in a WL mass model obtained using a linear Kaiser \& Squires (1993) mass reconstruction method with Gaussian smoothing (Figure 12). We then estimated redshifts for 12 peaks with nearby bright galaxies based on spectroscopic and photometric redshift information. Hectospec spectroscopic redshifts were available for 10 of the peaks. For the remaining galaxies, we used BPZ photometric redshifts derived using their $B_{\mathrm{J}} V_{\mathrm{J}} R_{\mathrm{C}} i^{\prime} z^{\prime}$ magnitudes. We note that these achieved a good accuracy of $\sim 3 \%(1+z)$ for the $\sim 300$ galaxies with $B_{\mathrm{J}} V_{\mathrm{J}} R_{\mathrm{C}} i^{\prime} z^{\prime}$ photometry and confident spectroscopic redshifts.

We identified six mass peaks coincident with bright galaxies in the background or foreground. We then eliminated those peaks from our mass model by setting the overdensity of those regions equal to zero (Figure 12) and rederived the mass profile as determined by WL. Based on fitting of NFW profiles to our SL and WL, we found that removal of these background structures lowered the virial mass $M_{\mathrm{vir}}$ by $\sim 7 \%$ and increased the concentration $c_{\text {vir }}$ by $\sim 5 \%$. (Fitting to $\mathrm{WL}$ alone yielded slightly higher $\sim 10 \%$ effects.) We conclude that background structures likely affect the mass and concentration measurements from joint SL+WL fitting at the $10 \%$ level or less. We made some attempt to maximize this effect by setting mass overdensities equal to zero (some overdensity should remain in these regions due to the cluster). However, our analysis was not extreme in either the number or sizes of areas eliminated.

\section{TRIAXIALITY FROM JOINT LENSING + X-RAY ANALYSIS}

Lensing analysis may yield higher mass estimates than X-ray analysis for either or both of the following reasons: (1) halo elongation and/or additional massive structures along the LOS boosting the lensing signal (Meneghetti et al. 2010; Newman et al. 2011; Morandi \& Limousin 2012) and (2) non-thermal gas pressure support (primarily turbulent flows and/or bulk motions) deviating from assumptions of hydrostatic equilibrium (HSE; Nagai et al. 2007; Lau et al. 2009; Kawaharada et al. 2010).

In cosmological simulations, dark matter halos are generally found to be prolate with typical axis ratios of $\sim 2: 1$. This elongation is generally found to decrease as a function of radius (Barnes \& Efstathiou 1987; Warren et al. 1992; Jing \& Suto 2002; Schulz et al. 2005; Lemze et al. 2012). This trend may be dampened by baryons, which are more dominant at smaller radii and act to make halos more spherical due to their collisional nature (e.g., Kazantzidis et al. 2004). Halo elongations along the LOS can bias both lensing strengths and cluster concentration measurements significantly high, such that the measured concentrations of a lensing-selected sample may be biased high by 50\%-100\% (Hennawi et al. 2007; Oguri \& Blandford 2009; Meneghetti et al. 2010, 2011).

Non-thermal pressures may account for $\sim 15 \%$ of the total support against gravitational collapse, thus biasing low by that amount the mass derived when assuming HSE (Nagai et al. 2007; Lau et al. 2009). In relaxed clusters, non-thermal pressure support is expected to increase with radius up to $\sim 30 \%-40 \%$ 
at the virial radius due to inflowing gas (Lau et al. 2009; Shaw et al. 2010; Cavaliere et al. 2011b). A possible minimum in the non-thermal pressure support at $\sim 0.1 r_{\text {vir }}$ has also been predicted (Molnar et al. 2010).

Previous joint lensing + X-ray analyses have allowed for these factors as global constants (e.g., Morandi et al. 2010, 2011b; Newman et al. 2011; Morandi \& Limousin 2012). Radial dependence of non-thermal pressure support was modeled by Morandi et al. (2011a). Here, we consider radial variation of this quantity and elongation. We only consider elongation along the LOS as our two-dimensional lens mass modeling already allows for elongation and more general asymmetries within the plane of the sky.

\subsection{Chandra X-Ray Observations and Analysis}

A2261 was observed by Chandra ACIS-I in programs 550 and 5007 (PI: Van Speybroeck) to depths of 9.0 and $24.3 \mathrm{ks}$, respectively (Morandi et al. 2007; Maughan et al. 2008; Gilmour et al. 2009; Mantz et al. 2010a).

We reprocessed and filtered the X-ray events in the latter observation in a standard manner using CIAO v4.3 and CALDB version 4.4.6. Based on $\sim 24,000$ net photon counts (0.7-7.0 keV), we extracted X-ray spectra within 15 annuli in the range $6^{\prime \prime}<R<3$ '.1 (20 kpc $\lesssim R \lesssim 650 \mathrm{kpc}$ ) centered on the X-ray peak, which is coincident with the center of the BCG. There were roughly equal net counts per annulus. A matched extraction of events from a reprojected, filtered, deep background events file was used for the background spectrum.

XMM observed A2261 on nine separate occasions between 2003 and 2004 for $\sim 12-13 \mathrm{ks}$ each. Each observation was heavily contaminated by proton flares and deemed unsuitable for analysis. It is likely that these lower priority observations were scheduled during periods of elevated particle backgrounds.

We fit the Chandra spectra simultaneously by creating models of hot gas in HSE in a dark matter NFW gravitational potential well using the JACO (Joint Analysis of Cluster Observations) software (Mahdavi et al. 2007). JACO allows for nuisance parameters such as an X-ray point source (none were detected) and contributions from a galactic soft background (found to be negligible in this case).

In Figure 13, we plot our NFW fit to the total mass (gas + dark matter) profile assuming a spherical halo and HSE. We fit out to $r=3 ! .1 \approx 667 \mathrm{kpc} h_{70}^{-1}$, or just beyond $r_{2500}=590 \mathrm{kpc}$ $h_{70}^{-1}$, corresponding to $M_{2500}=(0.29 \pm 0.05) \times 10^{15} M_{\odot} h_{70}^{-1}$, which we derive along with an NFW concentration $c_{2500}=$ $2.3 \pm 0.9$. This mass is $\sim 35 \%$ lower than the mass we derive at that radius based on our lensing analysis. For reference, if extrapolated to the virial radius, this profile would correspond to $M_{\text {vir }}=(0.82 \pm 0.14) \times 10^{15} M_{\odot} h_{70}^{-1}$ with $c_{\text {vir }}=9.1 \pm 3.0$. As we show, this is in good agreement with Zhang et al. (2010), who also fit out to larger radii using the $X M M$ data. Maughan et al. (2008) find a slightly larger $M_{500} \sim 0.80 \times 10^{15} M_{\odot}$ within $R_{500} \approx 1.31 \mathrm{Mpc}$ based on the Chandra data.

We also plot $20 \%$ deviations from HSE in the form of nonthermal pressure support. Though larger than expected within $r_{2500}$ (Lau et al. 2009; Shaw et al. 2010; Molnar et al. 2010; Cavaliere et al. 2011b; Nelson et al. 2012), this is what the data would require to bring the lensing and $\mathrm{X}$-ray masses derived in this work into agreement just within the error bars.

Mantz et al. (2010a) derive a higher gas mass than Zhang et al. (2010; also shown in Figure 13). Based on this, they derive a significantly higher $M_{500}=(1.44 \pm 0.26) \times 10^{15} M_{\odot}$ within

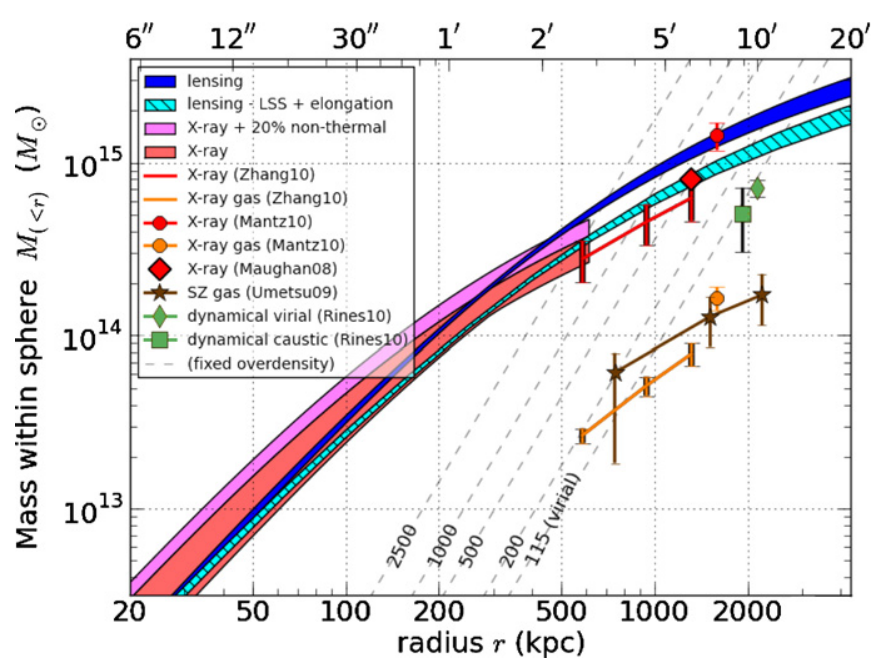

Figure 13. Mass profiles derived from various observational probes. We derive an X-ray mass (red curve, NFW profile) $\sim 35 \%$ lower than our lensing mass (blue, NFW profile) at $r_{2500} \sim 600 \mathrm{kpc}$. Marginal agreement can be achieved by allowing for $20 \%$ non-thermal pressure support (magenta), though this is much higher than generally expected at this radius (e.g., Lau et al. 2009). Agreement may be more readily achieved by an elongated halo with a 2:1 axis ratio for $r>100 \mathrm{kpc}$ (light blue hashed). However, the need for such elongation may be obviated completely by systematic uncertainties in the X-ray results (Maughan et al. 2008; Zhang et al. 2010; Mantz et al. 2010a). The latter result agrees well with our spherical lensing mass profile. A similar range of results was found by Conte et al. (2011), who consider various systematics. The dynamical mass estimates of Rines et al. (2010) are significantly lower $\left(M_{100} \sim(0.5-0.7) \times 10^{15} M_{\odot} h_{70}^{-1}\right)$. Also plotted are gas mass measurements based on X-ray (orange) and AMiBA SZE observations (brown stars; Umetsu et al. 2009).

(A color version of this figure is available in the online journal.)

$r_{500}=1.59 \pm 0.09 \mathrm{Mpc}$. This is in excellent agreement with our derived lensing mass.

Mantz et al. (2010a) assume a gas mass fraction $f_{\text {gas }} \sim 12 \%$ for A2261, very similar to the $f_{\text {gas }}$ derived by Zhang et al. (2010) assuming HSE. Various systematics are discussed further in Conte et al. (2011), who also derive a range of mass estimates for A2261 similar to that described already. We consider this full range in our analysis.

We note that published dynamical mass estimates of A2261 are significantly lower (Rines et al. 2010). These data are somewhat limited by bright stars in this field, hindering our ability to obtain additional spectra that might resolve this discrepancy.

X-ray observables, as well as the masses derived from them, are largely insensitive to halo elongation (e.g., Piffaretti et al. 2003; Gavazzi 2005; Buote \& Humphrey 2012a, 2012b), primarily because the intracluster gas is significantly less elongated due to dissipation (e.g., Lee \& Suto 2003). Masses derived from lensing data, however, may be strongly biased by halo elongation, as we discuss below.

\subsection{Halo Elongation}

As shown in Figure 13, our mass profiles derived independently from lensing and X-ray analyses are in good agreement in the core, while the latter exhibits a $\sim 35 \%$ deficit at the X-ray $r_{2500} \sim 600 \mathrm{kpc}$. This result is toward the low end of other X-ray mass estimates, so we consider this to be a limiting case. This deficit could best be accounted for by halo elongation along our LOS (though the Mantz et al. 2010a result would obviate the need for any such elongation). 
We found that an axis ratio of 2:1 is able to bring our lensing and X-ray results into better agreement at $r_{2500}$. This elongation is not required at inner radii where a spherical profile fits the data. Halo elongation is generally expected to decrease, not increase, with radius (e.g., Hayashi et al. 2007). However, we note that here we are probing the very inner core where the dense concentration of baryons may increase the sphericity (e.g., Kazantzidis et al. 2004). The large BCG of A2261 extends visibly to $r \sim 100 \mathrm{kpc}$.

We construct a toy model for the halo elongation $e=1-b / a$ varying with radius, increasing from 0 (spherical) for $r \leqslant 1 \mathrm{kpc}$ to 0.5 (an axis ratio of $2: 1$ ) beyond $r \geqslant 100 \mathrm{kpc}$. Between these two radii, it follows $e(r)=0.25 \log _{10}(r / \mathrm{kpc})$. The threedimensional mass density $\rho(r)$ scales with halo roundness $(\xi=1-e): \rho(r)=\xi(r) \rho_{\mathrm{NFW}}(u)$, where $u=\sqrt{x^{2}+y^{2}+(\xi z)^{2}}$ and $\rho_{\mathrm{NFW}}(u)=\rho_{s}\left(u / r_{s}\right)^{-1}\left(1+u / r_{s}\right)^{-2}$. This scaling preserves the projected mass density $\kappa(R)$ integrated along the LOS $(z$-axis) and thus preserves all lensing observables.

We applied this elongation profile to our primary joint (SL + WL shear + magnification) lensing profile NFW fit: $M_{\mathrm{vir}}=(2.2 \pm 0.2) \times 10^{15} M_{\odot} h_{70}^{-1}$ and $c_{\mathrm{vir}}=6.2 \pm 0.3$. We then calculated numerically the spherically averaged profile $M(<R)$ for this ellipsoidal mass distribution. This is plotted as the light blue hashed region in Figure 13. We find that this model agrees well with both our lensing and X-ray-derived mass profiles, whether including modest non-thermal pressure support or not.

A spherical NFW fit to this elongated profile yields $M_{\mathrm{vir}}=$ $(1.7 \pm 0.2) \times 10^{15} M_{\odot} h_{70}^{-1}$ and $c_{\mathrm{vir}}=4.6 \pm 0.2$. Applying the corrections for background/foreground LOS structures estimated in Section 6.1, we find $M_{\text {vir }} \sim 1.6 \times 10^{15} M_{\odot}(\mathrm{a} \sim 7 \%$ decrease $)$ and $c_{\text {vir }} \sim 4.8$ (a $\sim 5 \%$ increase $)$. Note that the former corrections for cluster halo elongation are significantly larger than those for LOS structure.

We also experimented with a more extreme (and less likely) axis ratio of $3: 1$ along the LOS as a further limiting case. We found that the spherically averaged outer mass profile $(R>500 \mathrm{kpc})$ dropped further, achieving better agreement with the Zhang et al. (2010) results. In this case we derive $M_{\text {vir }} \sim 1.3 \times 10^{15} M_{\odot}$ and $c_{\text {vir }} \sim 3.7$. We note that the inner mass profile drifted below our results unless we extended the sphericity of the inner halo out to $\sim 10 \mathrm{kpc}$ (previously $1 \mathrm{kpc}$ ).

This measurement method is consistent with that generally used to measure the mass profiles of simulated clusters. Enclosed mass (or, more often, density) is determined assuming spherical symmetry (and most often fit to an NFW profile) even though the halos are triaxial and asymmetric. And note that rather than attempting a noisy deprojection of the surface mass density (e.g., Saha \& Read 2009), we have instead solved the forward problem by projecting a spherical NFW profile to fit the lensing observables and then adding elongation to explore the degeneracies in the projected mass profile.

Another approach is to fit the lensing and X-ray observables to an ellipsoidal NFW profile, as in the Morandi \& Limousin (2012) analysis of A383, the first observed CLASH cluster. Notably, they allow for a fully general ellipsoidal gNFW (generalized NFW with variable inner slope) dark matter halo plus an exponential profile for the intracluster medium including nonthermal pressure support. Ideally, simulations will be analyzed in the same way allowing for direct comparisons. Until then, care must be taken when interpreting these results as the concentration and virial mass in these parameterizations may not correspond to the spherically averaged values.

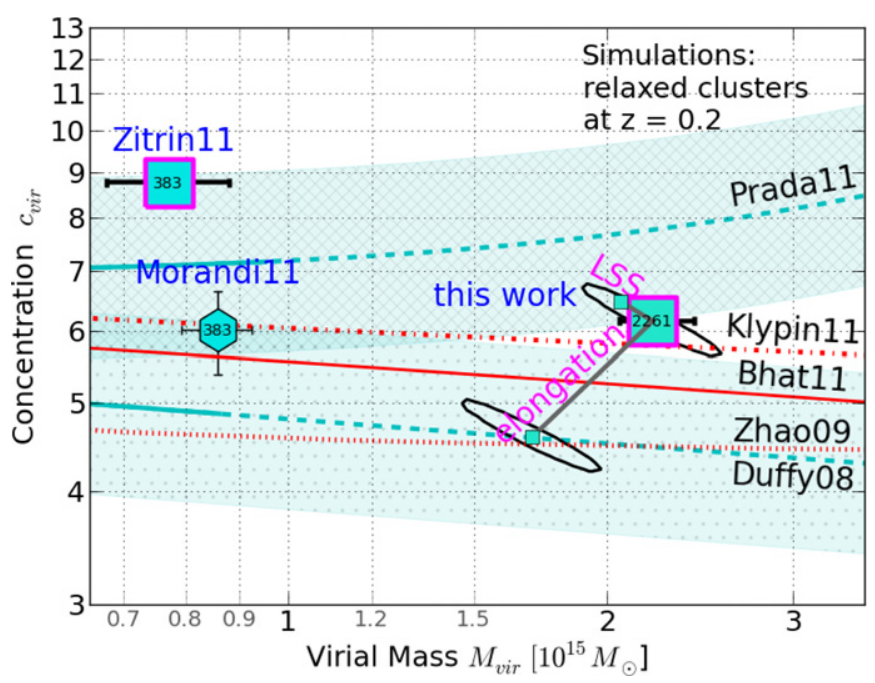

Figure 14. Observed virial masses $M_{\text {vir }}$ and concentrations $c_{\text {vir }}$ for CLASH clusters compared to the average $c(M, z)$ realized for relaxed clusters in simulations. Squares are from joint strong- + weak-lensing analyses of A2261 (this work) and A383 (Zitrin et al. 2011c). The hexagon is from Morandi \& Limousin (2012; A. Morandi \& M. Limousin 2011, private communication), who fit triaxial halos to A383 SL + X-ray data. For A2261, we plot both error bars $(1 \sigma$, marginalizing over the other parameter) and confidence contours $(1 \sigma)$. Systematic uncertainties are labeled: possible halo elongation (Section 7.2) and line-of-sight structures (Section 6.1). Results realized in two simulations (Duffy et al. 2008; Prada et al. 2012) are shown in light blue, including scatters of $\sim 0.1$ in $\log _{10}(c)(\sim 26 \%)$. Portions of these lines are dashed to indicate extrapolations to high masses where clusters are not realized in sufficient numbers. Average results from three additional simulations (Zhao et al. 2009; Klypin et al. 2011; Bhattacharya et al. 2011) are shown in red with styles solely for clarity. Results are plotted for relaxed cluster subsamples as determined by Duffy et al. (2008) and Bhattacharya et al. (2011), yielding concentrations $\sim 10 \%$ higher than for the full populations. This $10 \%$ factor is applied to the results from the other simulations.

(A color version of this figure is available in the online journal.)

Morandi \& Limousin (2012) derive spherically averaged $M_{\mathrm{vir}}=(8.6 \pm 0.7) \times 10^{14} M_{\odot}$ and $c_{\mathrm{vir}}=6.0 \pm 0.6(\mathrm{~A}$. Morandi \& M. Limousin 2011, private communication) based on joint SL + X-ray analysis of A383. We compare this to the $M_{\mathrm{vir}}=(7.7 \pm 1.0$ (stat.) \pm 0.4 (syst. $\left.)\right) \times 10^{14} M_{\odot}$ and $c_{\text {vir }}=8.8 \pm 0.4$ (stat.) \pm 0.2 (syst.) found by Zitrin et al. (2011c), who fit a spherical NFW profile to joint SL + WL constraints. The effect of correcting for elongation is to decrease the derived concentration as in our analysis of A2261 (see Figure 14).

\section{MASS PROFILE COMPARED TO SIMULATED CLUSTERS}

Based on our joint SL $+\mathrm{WL}+\mathrm{X}$-ray analysis, we find that A2261 is not significantly overconcentrated compared to simulated relaxed clusters of similar mass and redshift. This is demonstrated in Figure 14. Our range of allowed concentrations $(4.4 \lesssim c \lesssim 6.5)$ span the low end of average expectations $(4.5 \lesssim\langle c\rangle \lesssim 7.8)$ from simulations (Duffy et al. 2008; Zhao et al. 2009; Klypin et al. 2011; Prada et al. 2012; Bhattacharya et al. 2011). Results are also plotted for the first CLASH cluster A383 (Zitrin et al. 2011c; Morandi \& Limousin 2012).

Note that the recent Bolshoi and Multidark simulations (Klypin et al. 2011; Prada et al. 2012) produce halos with significantly higher concentrations than previous simulations (Neto et al. 2007; Macciò et al. 2008; Duffy et al. 2008; Zhao et al. 2009; although see Hennawi et al. 2007). Prada et al. (2012) find upturns in $c(M, z)$ for high masses and redshifts. This behavior is unexpected, and its origin needs to be understood. 


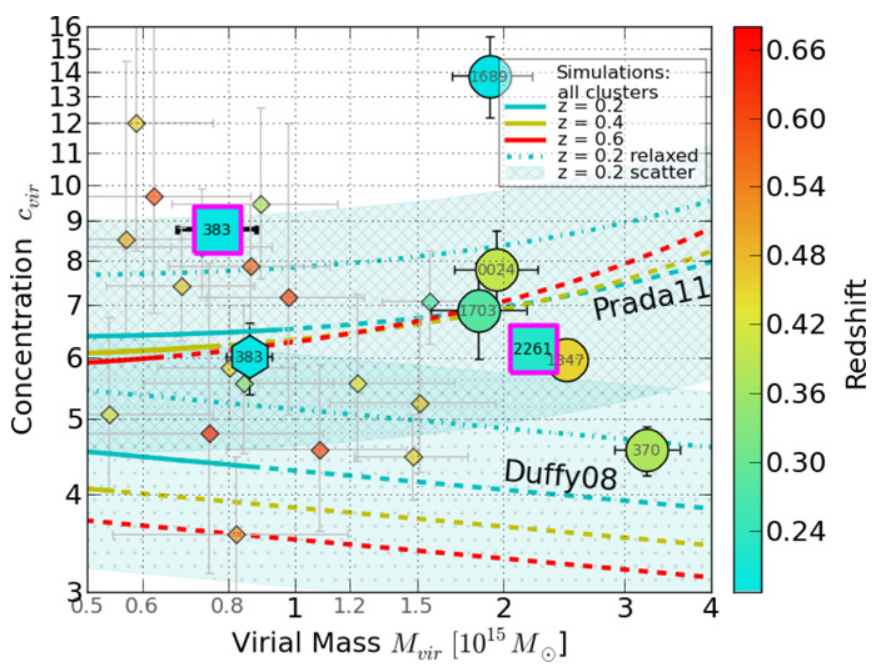

Figure 15. Similar to Figure 14 but now for all clusters (not just relaxed). The additional data points are all analyses of non-CLASH, lensing-biased clusters, as follows and all colored according to redshift. Circles are from Umetsu et al. (2011b) SL+WL analyses, and small diamonds are from Oguri et al. (2012) analyses with WL + basic SL constraints (the Einstein radii). Note that the average predictions from simulations for $c(M, z)$ for all clusters are $\sim 10 \%$ lower than for relaxed clusters. The expected scatters are larger: $\sim 0.15$ in $\log _{10}(c)(\sim 41 \%)$.

(A color version of this figure is available in the online journal.)

Bhattacharya et al. (2011) find no evidence for such upturns in their analysis of an even larger simulation, $2 \mathrm{Gpc} h^{-1}$ on a side, eight times the volume of Multidark, with the same number of particles $\left(2048^{3}\right)$.

A383 and A2261 are 2 of 20 CLASH clusters selected based on X-ray properties. We expect this sample to be less biased toward elongations along the LOS than a lensing-selected sample. However, some bias may remain. If we assume that all clusters are prolate and elongated in some direction, perhaps clusters that are roughly round and symmetric in the $\mathrm{X}$-rays may preferentially be elongated along our LOS. We will continue to quantify halo elongations and their effects on derived concentrations both in our observed CLASH clusters and in simulated clusters chosen to mimic the CLASH selection function.

A2261 is borderline relaxed. Gilmour et al. (2009) classified it as disturbed, but the X-ray peak is well aligned with the $\mathrm{BCG}$, and the X-ray luminosity is symmetric except for a subclump to the SW. Maughan et al. (2008) measured centroids in various annuli and found the rms shift to be modest $\langle w\rangle=$ $(7.1 \pm 0.6) \times 10^{-3} R_{500}$, consistent with that found for relaxed clusters $\langle w\rangle \lesssim 0.012 R_{500}$.

In Figure 15, we plot the expected $c(M, z)$ for all clusters versus the most robust results from other $\mathrm{SL}+\mathrm{WL}$ analyses to date, including those just mentioned plus Umetsu et al. (2011b) and Oguri et al. (2012). These clusters were initially selected for study based on their lensing strength; thus, their concentrations are expected to be biased significantly high. Disparity in this comparison is further increased as the expectations from simulations are lower. Average concentrations for relaxed clusters (as plotted in Figure 14) are found to be $\sim 10 \%$ higher (and have lower scatter) than averages for the general population as plotted in Figure 15.

\section{DISCUSSION: THE FORMATION TIME OF A2261}

We have found the mass profile and concentration of A2261 to be in agreement with values realized in cosmological sim-
Table 5

A2261 Formation Redshift $z_{f}$ Estimates Based on Various Criteria for "Formation"

\begin{tabular}{lccc}
\hline \hline & Redshift $z_{f}$ (Age of the Universe, Gyr) & \\
\hline$c_{\text {vir }}=11.1^{a}$ & $c_{\text {vir }}=6.3^{b}$ & $c_{\text {vir }}=4.6^{c}$ & $c_{1}{ }^{d}$ \\
\hline $2.9(2.2)$ & $1.2(5.0)$ & $0.6(7.8)$ & 3.5 \\
$2.3(2.8)$ & $0.9(6.2)$ & $0.4(9.2)$ & 4.1 \\
$1.7(3.8)$ & $0.5(8.4)$ & $0.13(11.8)$ & 5.1 \\
\hline
\end{tabular}

Notes. Based on $c_{\text {vir }} \approx c_{1}\left(1+z_{f}\right) /\left(1+z_{\mathrm{obs}}\right)$

${ }^{a}$ Umetsu et al. (2009) result.

b This work: spherical halo.

c This work: elongated halo.

d Normalization according to each definition, respectively, Cavaliere et al. (2011a), Wechsler et al. (2002), and Sadeh \& Rephaeli (2008).

ulations for similar clusters. This is contrary to the previous finding of Umetsu et al. (2009) based solely on ground-based data, which found a high concentration, suggesting an early formation time.

We can attempt to quantify this statement based on the relation $c_{\mathrm{obs}} \approx c_{1} a_{\mathrm{obs}} / a_{f}$ found in previous work (Bullock et al. 2001; Wechsler et al. 2002; Zhao et al. 2003; Wechsler et al. 2006). Here, $a=(1+z)^{-1}$ is the cosmic scale factor. The halo concentration is imprinted with the background density at its formation time via $c\left(z_{f}\right) \approx c_{1}\left(1+z_{f}\right)$ and then increases over time roughly as $c(z) \propto a=(1+z)^{-1}$.

The constant $c_{1}$ depends on the criteria used to define "formation time." Various definitions have been proposed based on the slowing rate of mass accretion (e.g., Wechsler et al. 2002; Cavaliere et al. 2011a) or mass attaining some fraction of the observed mass (e.g., Sadeh \& Rephaeli 2008).

We present results based on these various definitions in Table 5. Regardless of the definition, we note that the Umetsu et al. (2009) result of $c_{\text {vir }} \sim 11$ implies a formation time $\left(1.7 \lesssim z_{f} \lesssim 2.9\right)$ several billion years earlier than our primary result for a spherical halo $c_{\text {vir }} \sim 6.3\left(0.5 \lesssim z_{f} \lesssim 1.2\right)$. This, in turn, implies a formation time several billion years earlier than our result for an elongated halo $c_{\text {vir }} \sim 4.6\left(0.13 \lesssim z_{f} \lesssim 0.6\right)$. The lone $z_{f}<z_{\text {obs }}=0.225$ result would suggest that the cluster has yet to finish "forming" according to the Sadeh \& Rephaeli (2008) definition.

Concentration may be the observable most tightly correlated with age for relaxed clusters (Wong \& Taylor 2011), but other probes may also be brought to bear. Smith et al. (2010) studied BCG morphology, luminosity gap $\Delta m_{12}$ between the brightest and second-brightest cluster galaxy, substructure fraction $f_{\text {sub }}$, and cool core strength, as well as concentrations (as available from X-ray analyses in Sanderson et al. 2009) in a sample of 59 massive clusters, including A2261. A2261 was found to be one of four "fossil clusters" with a large luminosity gap $\Delta m_{12}>2$. Clusters with $\Delta m_{12} \gtrsim 1$ were found to have less substructure, stronger cool cores, and higher mass concentrations, all likely signatures of earlier formation times without recent major mergers. In these clusters, the BCG has presumably had time to grow and accrete a significant fraction of the substructure mass (see also Ascaso et al. 2011).

Based on X-ray observations, A2261 is borderline relaxed (see discussion in Section 8) and a borderline cool core cluster. Though the temperature profile dips down in the core (Cavagnolo et al. 2009), the central entropy floor $\left(K_{0}=61 \pm\right.$ $\left.8 \mathrm{keV} \mathrm{cm}{ }^{2}\right)$ is higher and the density profile slope $(\alpha \sim-0.7$ at $\left.0.04 r_{500}\right)$ shallower than generally found $\left(K_{0}<30 \mathrm{keV} \mathrm{cm}{ }^{2}\right.$ 
and $\alpha \lesssim-0.85$ ) for cool core clusters (Sanderson et al. 2009; Smith et al. 2010). There is no obvious star formation visible in the near-UV/optical as often found in cool core clusters. A radio source aligned with the BCG is detected with $\sim 5.3 \mathrm{mJy}$ $\sim 8 \times 10^{23} \mathrm{~W} \mathrm{~Hz}^{-1}$ at $1.4 \mathrm{GHz}$ in NVSS (Condon et al. 1998) and $3.39 \mathrm{mJy}$ at $21 \mathrm{~cm}$ in FIRST (Becker et al. 1995). All 69 radio-bright $\left(>2 \times 10^{23} \mathrm{~W} \mathrm{~Hz}^{-1}\right.$ at $\left.1.4 \mathrm{GHz}\right)$ BCGs analyzed by Sun (2009) were found to be in X-ray cool cores.

Ultimately, analyses of these various observables in CLASH clusters and in simulated clusters with similar properties will contribute to significant advancements in our understanding of structure formation and evolution.

\section{CONCLUSIONS}

We performed the first robust joint SL and WL analysis of the galaxy cluster A2261. We find a halo virial mass $M_{\mathrm{vir}}=$ $(2.2 \pm 0.2) \times 10^{15} M_{\odot} h_{70}^{-1}$ and concentration $c_{\mathrm{vir}}=6.2 \pm 0.3$ when assuming a spherical halo. These tight constraints were enabled through a combination of the 16-band imaging from CLASH with multiband wide-field imaging from the Subaru and KPNO telescopes. The results show that A2261 is not "overconcentrated" as previously found but rather is in good agreement with predictions from $\Lambda \mathrm{CDM} N$-body simulations.

To explore halo elongation along the LOS, we also derived a mass profile based on Chandra X-ray data, finding it to be $\sim 35 \%$ below the lensing mass profile at $r_{2500}(\sim 500 \mathrm{kpc})$. This deficit may be explained by an axis ratio of $\sim 2: 1$ outside the inner core $r \sim 100 \mathrm{kpc}$, corresponding to the visible extent of the BCG. This elongated mass profile has a lower spherically defined virial mass $M_{\mathrm{vir}}=1.65_{-0.12}^{+0.16} \times 10^{15} M_{\odot} h_{70}^{-1}$ and concentration $c_{\text {vir }}=4.6 \pm 0.2$. Correcting for the lensing effects of massive background structures may increase $c_{\text {vir }}$ by $\sim 5 \%$ and decrease $M_{\text {vir }}$ by $\sim 7 \%$. This lower $c_{\text {vir }} \sim 4.8$ still agrees with predictions from many simulations but is lower than predicted by one recent study (Prada et al. 2012).

The need to assume halo elongation is critically tied to the reliability of the X-ray mass profile. Non-thermal pressure support may account partially for the lower X-ray mass. Published $\mathrm{X}$-ray mass estimates have significant scatter, including one result in excellent agreement with our spherical lensing mass at $r_{500}(\sim 1.6 \mathrm{Mpc})$.

The CLASH survey is providing fundamental and substantial improvements in the quantity and quality of observational constraints on cluster dark matter halos. Simulations will be tasked with reproducing these empirical results, contributing significantly to our understanding of structure formation. Ultimately our results will either confirm $\Lambda \mathrm{CDM}$ predictions or perhaps yield clues as to the nature of dark energy.

We thank Nobuhiro Okabe for providing his weak-lensing shape measurement catalog for comparison with this work. We also thank Nobuhiro, Mark Voit, Jack Sayers, and Nicole Czakon for useful discussions. Portions of this collaborative effort were carried out at the Keck Institute for Space Studies, who we thank for their hospitality. We thank Perry Berlind and Mike Calkins for efficient operation of the Hectospec, and we thank Susan Tokarz for running the Hectospec data reduction pipeline. We also thank the referee for excellent detailed comments that significantly improved the manuscript.

The CLASH Multi-Cycle Treasury Program is based on observations made with the NASA/ESA Hubble Space Telescope. The Space Telescope Science Institute is operated by the
Association of Universities for Research in Astronomy, Inc., under NASA contract NAS 5-26555. ACS was developed under NASA Contract NAS 5-32864.

This research is supported in part by NASA Grant HSTGO-12065.01-A, National Science Council of Taiwan Grant NSC100-2112-M-001-008-MY3, the Israel Science Foundation, the German Science Foundation (Transregio TR 33), Spanish MICINN Grant YA2010-22111-C03-00, funding from the Junta de Andalucía Proyecto de Excelencia NBL2003, INAF Contracts ASI-INAF I/009/10/0, ASI-INAF I/023/ 05/0, ASI-INAF I/088/06/0, PRIN INAF 2009, and PRIN INAF 2010, NSF CAREER Grant AST-0847157, the UK's STFC, the Royal Society, and the Wolfson Foundation. K.U. acknowledges support from the Academia Sinica Career Development Award. A.Z. is supported by contract research Internationale Spitzenforschung II-1 of the Baden-Württemberg Stiftung. M.J.G., K.J.R., A.D., and M.J.K. acknowledge partial support from the Smithsonian Institution. K.J.R. was funded in part by a Cottrell College Science Award from the Research Corporation. A.D. gratefully acknowledges partial support from the INFN Grant PD51 and the PRIN-MIUR-2008 Grant 2008NR3EBK_003 "Matter-antimatter asymmetry, dark matter and dark energy in the LHC era." L.I. acknowledges support from a Conicyt FONDAP/BASAL grant. P.R. and S.S. acknowledge support from the DFG cluster of excellence Origin and Structure of the Universe program.

Facilities: HST (ACS, WFC3), Subaru (Suprime-Cam), Mayall, CXO (ACIS), MMT (Hectospec)

\section{REFERENCES}

Abazajian, K. N., Adelman-McCarthy, J. K., Agüeros, M. A., et al. 2009, ApJS, 182,543

Allen, S. W., Evrard, A. E., \& Mantz, A. B. 2011, ARA\&A, 49, 409

Ascaso, B., Aguerri, J. A. L., Varela, J., et al. 2011, ApJ, 726, 69

Bahé, Y. M., McCarthy, I. G., \& King, L. J. 2012, MNRAS, 421, 1073

Baldi, M. 2012, MNRAS, 420, 430

Barnes, J., \& Efstathiou, G. 1987, ApJ, 319, 575

Becker, M. R., \& Kravtsov, A. V. 2011, ApJ, 740, 25

Becker, R. H., White, R. L., \& Helfand, D. J. 1995, ApJ, 450, 559

Benítez, N. 2000, ApJ, 536, 571

Benítez, N., Ford, H., Bouwens, R., et al. 2004, ApJS, 150, 1

Benson, B. A., de Haan, T., Dudley, J. P., et al. 2011, arXiv:1112.5435

Bertin, E., \& Arnouts, S. 1996, A\&AS, 117, 393

Bhattacharya, S., Habib, S., \& Heitmann, K. 2011, arXiv:1112.5479

Borgani, S., \& Kravtsov, A. 2011, Adv. Sci. Lett., 4, 204

Broadhurst, T., Benítez, N., Coe, D., et al. 2005a, ApJ, 621, 53

Broadhurst, T., Takada, M., Umetsu, K., et al. 2005b, ApJ, 619, L143

Broadhurst, T., Umetsu, K., Medezinski, E., Oguri, M., \& Rephaeli, Y. 2008, ApJ, 685, L9

Broadhurst, T. J., \& Barkana, R. 2008, MNRAS, 390, 1647

Broadhurst, T. J., Taylor, A. N., \& Peacock, J. A. 1995, ApJ, 438, 49

Brown, W. R., Geller, M. J., Kenyon, S. J., \& Diaferio, A. 2010, AJ, 139, 59

Bryan, G. L., \& Norman, M. L. 1998, ApJ, 495, 80

Bullock, J. S., Kolatt, T. S., Sigad, Y., et al. 2001, MNRAS, 321, 559

Buote, D. A., \& Humphrey, P. J. 2012a, MNRAS, 420, 1693

Buote, D. A., \& Humphrey, P. J. 2012b, MNRAS, 421, 1399

Capak, P., Aussel, H., Ajiki, M., et al. 2007, ApJS, 172, 99

Carlesi, E., Knebe, A., Yepes, G., et al. 2011, MNRAS, 418, 2715

Cavagnolo, K. W., Donahue, M., Voit, G. M., \& Sun, M. 2009, ApJS, 182, 12

Cavaliere, A., Lapi, A., \& Fusco-Femiano, R. 2011a, ApJ, 742, 19

Cavaliere, A., Lapi, A., \& Fusco-Femiano, R. 2011b, A\&A, 525, A110

Chongchitnan, S., \& Silk, J. 2011, Phys. Rev. D, 83, 083504

Clowe, D., Bradač, M., Gonzalez, A. H., et al. 2006, ApJ, 648, L109

Clowe, D., Luppino, G. A., Kaiser, N., \& Gioia, I. M. 2000, ApJ, 539, 540

Coe, D. 2010, arXiv:1005.0411

Coe, D., Benítez, N., Broadhurst, T., \& Moustakas, L. A. 2010, ApJ, 723, 1678

Coe, D., Benítez, N., Sánchez, S. F., et al. 2006, AJ, 132, 926

Coe, D., Fuselier, E., Benítez, N., et al. 2008, ApJ, 681, 814

Condon, J. J., Cotton, W. D., Greisen, E. W., et al. 1998, AJ, 115, 1693 
Conte, A., de Petris, M., Comis, B., Lamagna, L., \& de Gregori, S. 2011, A\&A, 532, A 14

Crawford, C. S., Edge, A. C., Fabian, A. C., et al. 1995, MNRAS, 274, 75

D’Aloisio, A., \& Natarajan, P. 2012, arXiv:1202.0553

Davis, O., Devriendt, J., Colombi, S., Silk, J., \& Pichon, C. 2011, MNRAS, 413, 2087

Diaferio, A. 2009, arXiv:0901.0868

Duffy, A. R., Schaye, J., Kay, S. T., \& Dalla Vecchia, C. 2008, MNRAS, 390, L64

Duffy, A. R., Schaye, J., Kay, S. T., et al. 2010, MNRAS, 405, 2161

Einasto, J. 1965, Trudy Inst. Astroz. Alma-Ata, 51, 87

Eisenhardt, P. R. M., Brodwin, M., Gonzalez, A. H., et al. 2008, ApJ, 684, 905

Fabricant, D., Fata, R., Roll, J., et al. 2005, PASP, 117, 1411

Fahlman, G., Kaiser, N., Squires, G., \& Woods, D. 1994, ApJ, 437, 56

Fedeli, C. 2012, MNRAS, 424, 1244

Fedeli, C., \& Bartelmann, M. 2007, A\&A, 461, 49

Fioc, M., \& Rocca-Volmerange, B. 1997, A\&A, 326, 950

Foley, R. J., Andersson, K., Bazin, G., et al. 2011, ApJ, 731, 86

Ford, H. C., Clampin, M., Hartig, G. F., et al. 2003, Proc. SPIE, 4854, 81

Francis, M. J., Lewis, G. F., \& Linder, E. V. 2009, MNRAS, 394, 605

Gao, L., Navarro, J. F., Cole, S., et al. 2008, MNRAS, 387, 536

Gavazzi, R. 2005, A\&A, 443, 793

Geller, M. J., Dell'Antonio, I. P., Kurtz, M. J., et al. 2005, ApJ, 635, L125

Gilmour, R., Best, P., \& Almaini, O. 2009, MNRAS, 392, 1509

Gobat, R., Daddi, E., Onodera, M., et al. 2011, A\&A, 526, A133

Grossi, M., \& Springel, V. 2009, MNRAS, 394, 1559

Gruen, D., Bernstein, G. M., Lam, T. Y., \& Seitz, S. 2011, MNRAS, 416, 1392

Harrison, I., \& Coles, P. 2011, MNRAS, 418, L20

Harrison, I., \& Coles, P. 2012, MNRAS, 421, L19

Hayashi, E., Navarro, J. F., \& Springel, V. 2007, MNRAS, 377, 50

Hennawi, J. F., Dalal, N., Bode, P., \& Ostriker, J. P. 2007, ApJ, 654, 714

Henry, J. P., Evrard, A. E., Hoekstra, H., Babul, A., \& Mahdavi, A. 2009, ApJ, 691,1307

Heymans, C., Van Waerbeke, L., Bacon, D., et al. 2006, MNRAS, 368, 1323

Hoekstra, H., Hartlap, J., Hilbert, S., \& van Uitert, E. 2011, MNRAS, 412, 2095

Host, O. 2012, MNRAS, 420, L18

Hotchkiss, S. 2011, J. Cosmol. Astropart. Phys., JCAP07(2011)004

Hoyle, B., Jimenez, R., Verde, L., \& Hotchkiss, S. 2012, J. Cosmol. Astropart. Phys., JCAP02(2012)009

Huang, X., Morokuma, T., Fakhouri, H. K., et al. 2009, ApJ, 707, L12

Ilbert, O., Capak, P., Salvato, M., et al. 2009, ApJ, 690, 1236

Jee, M. J., Dawson, K. S., Hoekstra, H., et al. 2011, ApJ, 737, 59

Jee, M. J., Rosati, P., Ford, H. C., et al. 2009, ApJ, 704, 672

Jing, Y. P. 2000, ApJ, 535, 30

Jing, Y. P., \& Suto, Y. 2002, ApJ, 574, 538

Jullo, E., Kneib, J.-P., Limousin, M., et al. 2007, New J. Phys., 9, 447

Jullo, E., Natarajan, P., Kneib, J.-P., et al. 2010, Science, 329, 924

Kaiser, N. 1995, ApJ, 439, L1

Kaiser, N., \& Squires, G. 1993, ApJ, 404, 441

Kaiser, N., Squires, G., \& Broadhurst, T. 1995, ApJ, 449, 460

Kassiola, A., \& Kovner, I. 1993, ApJ, 417, 450

Kawaharada, M., Okabe, N., Umetsu, K., et al. 2010, ApJ, 714, 423

Kazantzidis, S., Kravtsov, A. V., Zentner, A. R., et al. 2004, ApJ, 611, L73

Keeton, C. R., \& Moustakas, L. A. 2009, ApJ, 699, 1720

Kimble, R. A., MacKenty, J. W., O’Connell, R. W., \& Townsend, J. A. 2008, Proc. SPIE, 7010, 70101E

Klypin, A. A., Trujillo-Gomez, S., \& Primack, J. 2011, ApJ, 740, 102

Kneib, J.-P. 1993, PhD thesis, Université Paul Sabatier, Toulouse

Koekemoer, A. M., Faber, S. M., Ferguson, H. C., et al. 2011, ApJS, 197, 36

Kurtz, M. J., \& Mink, D. J. 1998, PASP, 110, 934

Lau, E. T., Kravtsov, A. V., \& Nagai, D. 2009, ApJ, 705, 1129

Lauer, T. R. 1986, ApJ, 311, 34

Lee, J., \& Suto, Y. 2003, ApJ, 585, 151

Lemze, D., Sadeh, S., \& Rephaeli, Y. 2009, MNRAS, 397, 1876

Lemze, D., Wagner, R., Rephaeli, Y., et al. 2012, ApJ, 752, 141

Macciò, A. V., Dutton, A. A., \& van den Bosch, F. C. 2008, MNRAS, 391, 1940

Mahdavi, A., Hoekstra, H., Babul, A., et al. 2007, ApJ, 664, 162

Mantz, A., Allen, S. W., Ebeling, H., Rapetti, D., \& Drlica-Wagner, A. 2010a, MNRAS, 406, 1773

Mantz, A., Allen, S. W., Rapetti, D., \& Ebeling, H. 2010b, MNRAS, 406, 1759

Massey, R., Rhodes, J., Ellis, R., et al. 2007, Nature, 445, 286

Maughan, B. J., Jones, C., Forman, W., \& Van Speybroeck, L. 2008, ApJS, 174, 117

Mead, J. M. G., King, L. J., Sijacki, D., et al. 2010, MNRAS, 406, 434

Medezinski, E., Broadhurst, T., Umetsu, K., Benítez, N., \& Taylor, A. 2011, MNRAS, 414, 1840

Medezinski, E., Broadhurst, T., Umetsu, K., et al. 2007, ApJ, 663, 717
Medezinski, E., Broadhurst, T., Umetsu, K., et al. 2010, MNRAS, 405, 257

Meneghetti, M., Fedeli, C., Pace, F., Gottlöber, S., \& Yepes, G. 2010, A\&A, 519, A90

Meneghetti, M., Fedeli, C., Zitrin, A., et al. 2011, A\&A, 530, A17

Merritt, D., Graham, A. W., Moore, B., Diemand, J., \& Terzić, B. 2006, AJ, 132,2685

Merritt, D., Navarro, J. F., Ludlow, A., \& Jenkins, A. 2005, ApJ, 624, L85

Merten, J., Coe, D., Dupke, R., et al. 2011, MNRAS, 417, 333

Mink, D. J., Wyatt, W. F., Caldwell, N., et al. 2007, in ASP Conf. Ser. 376, Astronomical Data Analysis Software and Systems XVI, ed. R. A. Shaw, F. Hill, \& D. J. Bell (San Francisco, CA: ASP), 249

Miyazaki, S., Komiyama, Y., Sekiguchi, M., et al. 2002, PASJ, 54, 833

Molnar, S. M., Chiu, I., Umetsu, K., et al. 2010, ApJ, 724, L1

Morandi, A., Ettori, S., \& Moscardini, L. 2007, MNRAS, 379, 518

Morandi, A., \& Limousin, M. 2012, MNRAS, 421, 3147

Morandi, A., Limousin, M., Sayers, J., et al. 2011a, arXiv:1111.6189

Morandi, A., Pedersen, K., \& Limousin, M. 2010, ApJ, 713, 491

Morandi, A., Pedersen, K., \& Limousin, M. 2011b, ApJ, 729, 37

Nagai, D., Vikhlinin, A., \& Kravtsov, A. V. 2007, ApJ, 655, 98

Natarajan, P., De Lucia, G., \& Springel, V. 2007, MNRAS, 376, 180

Navarro, J. F., Frenk, C. S., \& White, S. D. M. 1996, ApJ, 462, 563

Navarro, J. F., Hayashi, E., Power, C., et al. 2004, MNRAS, 349, 1039

Navarro, J. F., Ludlow, A., Springel, V., et al. 2010, MNRAS, 402, 21

Nelson, K., Rudd, D. H., Shaw, L., \& Nagai, D. 2012, ApJ, 751, 121

Neto, A. F., Gao, L., Bett, P., et al. 2007, MNRAS, 381, 1450

Newman, A. B., Treu, T., Ellis, R. S., \& Sand, D. J. 2011, ApJ, 728, L39

Nonino, M., Dickinson, M., Rosati, P., et al. 2009, ApJS, 183, 244

Oguri, M., Bayliss, M. B., Dahle, H., et al. 2012, MNRAS, 420, 3213

Oguri, M., \& Blandford, R. D. 2009, MNRAS, 392, 930

Oguri, M., Hennawi, J. F., Gladders, M. D., et al. 2009, ApJ, 699, 1038

Okabe, N., Takada, M., Umetsu, K., Futamase, T., \& Smith, G. P. 2010, PASJ, 62, 811

Papovich, C., Momcheva, I., Willmer, C. N. A., et al. 2010, ApJ, 716, 1503

Paranjape, A., Gordon, C., \& Hotchkiss, S. 2011, Phys. Rev. D, 84, 023517

Piffaretti, R., Jetzer, P., \& Schindler, S. 2003, A\&A, 398, 41

Planck Collaboration, Aghanim, N., Arnaud, M., Ashdown, M., et al. 2011, A\&A, 536, A26

Postman, M., Coe, D., Benítez, N., et al. 2012a, ApJS, 199, 25

Postman, M., Lauer, T. R., Donahue, M., et al. 2012b, arXiv:1205.3839

Prada, F., Klypin, A. A., Cuesta, A. J., Betancort-Rijo, J. E., \& Primack, J. 2012, MNRAS, 423, 3018

Reed, D. S., Koushiappas, S. M., \& Gao, L. 2011, MNRAS, 415, 3177

Richard, J., Smith, G. P., Kneib, J., et al. 2010, MNRAS, 404, 325

Rines, K., \& Diaferio, A. 2006, AJ, 132, 1275

Rines, K., \& Diaferio, A. 2010, AJ, 139, 580

Rines, K., Geller, M. J., \& Diaferio, A. 2010, ApJ, 715, L180

Rines, K., Geller, M. J., Kurtz, M. J., \& Diaferio, A. 2005, AJ, 130, 1482

Rosati, P., Tozzi, P., Gobat, R., et al. 2009, A\&A, 508, 583

Rozo, E., \& Schmidt, F. 2010, arXiv:1009.5735

Sadeh, S., \& Rephaeli, Y. 2008, MNRAS, 388, 1759

Saha, P., \& Read, J. I. 2009, ApJ, 690, 154

Sanderson, A. J. R., Edge, A. C., \& Smith, G. P. 2009, MNRAS, 398, 1698

Santos, J. S., Fassbender, R., Nastasi, A., et al. 2011, A\&A, 531, L15

Santos, J. S., Tozzi, P., Rosati, P., Nonino, M., \& Giovannini, G. 2012, A\&A, 539, A105

Schulz, A. E., Hennawi, J., \& White, M. 2005, Astropart. Phys., 24, 409

Schwope, A. D., Lamer, G., de Hoon, A., et al. 2010, A\&A, 513, L10

Sehgal, N., Trac, H., Acquaviva, V., et al. 2011, ApJ, 732, 44

Sereno, M., Jetzer, P., \& Lubini, M. 2010, MNRAS, 403, 2077

Serra, A. L., Angus, G. W., \& Diaferio, A. 2010, A\&A, 524, A16

Shaw, L. D., Nagai, D., Bhattacharya, S., \& Lau, E. T. 2010, ApJ, 725, 1452

Smith, G. P., Khosroshahi, H. G., Dariush, A., et al. 2010, MNRAS, 409, 169

Stanford, S. A., Romer, A. K., Sabirli, K., et al. 2006, ApJ, 646, L13

Sun, M. 2009, ApJ, 704, 1586

Umetsu, K., Birkinshaw, M., Liu, G., et al. 2009, ApJ, 694, 1643

Umetsu, K., \& Broadhurst, T. 2008, ApJ, 684, 177

Umetsu, K., Broadhurst, T., Zitrin, A., et al. 2011a, ApJ, 738, 41

Umetsu, K., Broadhurst, T., Zitrin, A., Medezinski, E., \& Hsu, L. 2011b, ApJ, 729,127

Umetsu, K., Medezinski, E., Broadhurst, T., et al. 2010, ApJ, 714, 1470

Umetsu, K., Medezinski, E., Nonino, M., et al. 2012, ApJ, 755, 56

Vikhlinin, A., Kravtsov, A. V., Burenin, R. A., et al. 2009, ApJ, 692, 1060

Waizmann, J.-C., Ettori, S., \& Moscardini, L. 2011, MNRAS, 418, 456

Waizmann, J.-C., Ettori, S., \& Moscardini, L. 2012a, MNRAS, 420, 1754

Waizmann, J.-C., Ettori, S., \& Moscardini, L. 2012b, MNRAS, 422, 3554

Warren, M. S., Quinn, P. J., Salmon, J. K., \& Zurek, W. H. 1992, ApJ, 399, 405 
Wechsler, R. H., Bullock, J. S., Primack, J. R., Kravtsov, A. V., \& Dekel, A. 2002, ApJ, 568, 52

Wechsler, R. H., Zentner, A. R., Bullock, J. S., Kravtsov, A. V., \& Allgood, B. 2006, ApJ, 652, 71

Wong, A. W. C., \& Taylor, J. E. 2011, arXiv:1112.4229

Wuyts, S., Labbé, I., Schreiber, N. M. F., et al. 2008, ApJ, 682, 985

Zhang, Y., Okabe, N., Finoguenov, A., et al. 2010, ApJ, 711, 1033

Zhao, D. H., Jing, Y. P., Mo, H. J., \& Börner, G. 2009, ApJ, 707, 354

Zhao, D. H., Mo, H. J., Jing, Y. P., \& Börner, G. 2003, MNRAS, 339, 12

Zitrin, A., \& Broadhurst, T. 2009, ApJ, 703, L132
Zitrin, A., Broadhurst, T., Barkana, R., Rephaeli, Y., \& Benítez, N. 2011a, MNRAS, 410, 1939

Zitrin, A., Broadhurst, T., Bartelmann, M., et al. 2012a, MNRAS, 423, 2308

Zitrin, A., Broadhurst, T., Coe, D., et al. 2011b, MNRAS, 413, 1753

Zitrin, A., Broadhurst, T., Coe, D., et al. 2011c, ApJ, 742, 117

Zitrin, A., Broadhurst, T., Rephaeli, Y., \& Sadeh, S. 2009a, ApJ, 707, L102

Zitrin, A., Broadhurst, T., Umetsu, K., et al. 2009b, MNRAS, 396, 1985

Zitrin, A., Broadhurst, T., Umetsu, K., et al. 2010, MNRAS, 408, 1916

Zitrin, A., Moustakas, J., Bradley, L., et al. 2012b, ApJ, 747, L9

Zitrin, A., Rosati, P., Nonino, M., et al. 2012c, ApJ, 749, 97 